\title{
ISOPHOTAL SHAPES OF EARLY-TYPE GALAXIES TO VERY FAINT LEVELS
}

\author{
Laxmikant Chaware $^{1}$, Russell Cannon ${ }^{2}$, Ajit K. Kembhavi ${ }^{3}$, Ashish Mahabal ${ }^{4}$, and S. K. Pandey ${ }^{1}$ \\ ${ }^{1}$ School of Studies in Physics, Pt. Ravishankar Shukla University, Raipur 492010, India \\ 2 Australian Astronomical Observatory, P.O. Box 915, North Ryde, NSW 1670, Australia \\ ${ }^{3}$ Inter University Center for Astronomy and Astrophysics, Pune, India \\ ${ }^{4}$ California Institute of Technology, Pasadena, CA 91125, USA \\ Received 2013 June 2; accepted 2014 March 31; published 2014 May 8
}

\begin{abstract}
We report on a study of the isophotal shapes of early-type galaxies to very faint levels, reaching $\sim 0.1 \%$ of the sky brightness. The galaxies are from the Large Format Camera (LFC) fields obtained using the Palomar $5 \mathrm{~m}$ Hale Telescope, with integrated exposures ranging from 1 to $4 \mathrm{hr}$ in the Sloan Digital Sky Survey $r, i$, and $z$ bands. The shapes of isophotes of early-type galaxies are important, as they are correlated with the physical properties of the galaxies and are influenced by galaxy formation processes. In this paper, we report on a sample of $132 \mathrm{E}$ and SO galaxies in one LFC field. We have redshifts for 53 of these, obtained using AAOmega on the Anglo-Australian Telescope. The shapes of early-type galaxies often vary with radius. We derive average values of isophotal shape parameters in four different radial bins along the semi-major axis in each galaxy. We obtain empirical fitting formulae for the probability distribution of the isophotal parameters in each bin and investigate for possible correlations with other global properties of the galaxies. Our main finding is that the isophotal shapes of the inner regions are statistically different from those in the outer regions. This suggests that the outer and inner parts of early-type galaxies have evolved somewhat independently.
\end{abstract}

Key words: galaxies: elliptical and lenticular, $\mathrm{cD}$ - galaxies: photometry - galaxies: statistics - galaxies: structure

Online-only material: color figures, machine-readable tables

\section{INTRODUCTION}

The morphology and colors of galaxies carry important information about their formation and evolution. Bender et al. (1988) studied a sample of 109 large, nearby, early-type galaxies to examine their isophotal shapes, using Fourier expansions in the polar angle to distinguish between boxy (rectangular) isophotes and disky (pointed) isophotes. Studies have shown that elliptical galaxies with disky isophotes tend to be fainter, rotationally supported, lack X-ray and radio activity, and have power-law nuclear light profiles, while those with boxy isophotes tend to be brighter, supported by random motions, have significant $\mathrm{X}$-ray and radio activity, and a peaked nuclear profile (Ferrarese et al. 1994; van den Bosch 1994; Rest et al. 2001; Lauer 2005). The shapes of galaxies may also be correlated with their ages (Ryden et al. 2001). Recently, Hao et al. (2006a) studied the isophotal shapes of a large sample of 847 early-type galaxies from the Sloan Digital Sky Survey (SDSS), and Pasquali et al. (2007) have used the same sample to investigate the dependence of the isophotal structure on the active galactic nucleus activity and environment of the galaxies. The dynamic range available to these studies is limited, due to the short exposure of the SDSS. The isophotal shape study by Bender et al. (1988) extends to 1.5 times effective radius $\left(1.5 r_{e}\right)$ of the galaxies, while Hao et al. (2006a) studied their larger sample of 857 nearby galaxies with redshift $z<0.05$ extending to 1.5 times the Petrosian half-light radius $\left(1.5 R_{50}\right)$.

In this paper, we revisit the properties of isophotal shapes for a sample of more distant early-type galaxies in a single field, using exceptionally deep CCD exposures. These enable us to go out to much larger radii of $\sim 4.5 R_{50}$ and reach $\sim 4 \mathrm{mag} \mathrm{arcsec}^{-2}$ deeper in surface brightness (SB). We have obtained redshifts for a subsample of these galaxies: the redshifts peak at $z \sim 0.1$ and extend to $z \sim 0.8$, covering a wide range of absolute magnitudes.
Studies of the faint outer parts of galaxies are not new. By 1980, Malin (e.g., Malin \& Carter 1980) was using sky survey photographs to create images that reached 10 mag below the level of the sky background. These led to the discovery of very faint shells and tails around galaxies, soon interpreted as evidence for dynamical evolution due to gravitational interactions and mergers (e.g., Quinn 1984). Capaccioli et al. (1988) combined photographic and CCD imaging of nine nearby galaxies, to explore the boundary between E and S0 galaxies by looking at the changing shapes of isophotes with radius.

We extend the methodology of Bender et al. (1988) and derive mean isophote parameters for up to four radial bins for each galaxy. Pointed isophotes can usually be attributed to a galaxy which consists of a spheroid and a weak disk, which is being viewed more or less edge-on. Nieto \& Bender (1989) suggest that some pointed isophotes may be due to tidal extensions. But the origin of boxy isophotes is still unclear. Naab et al. (1999) showed that mergers of spiral galaxies with comparable mass are more likely to form boxy ellipticals, while mergers of spiral galaxies with differing mass can lead to disky ellipticals. However, disk-disk mergers do not form a perfect dichotomy (Nabb \& Burkert 2003), while elliptical-elliptical mergers always lead to boxy isophotes (Nabb et al. 2006). Using $\mathrm{N}$-body simulation, Bournaud et al. (2005) found that for a 7:1 merger, isophotes in the inner region (bulge) are boxy, while the outer isophotes are disky. Such radial variation is also seen in the analysis of Two Micron All Sky Survey (2MASS) data for Arp mergers by Chitre \& Jog (2002). Our study of isophotal shapes to faint levels will be useful for better comparison between observations and such theoretical possibilities.

This paper is organized in the following way: in Section 2, we describe the imaging data, sample selection, bulge-disk decomposition, and spectroscopic data used for this study. In Section 3, surface photometry of sample galaxies, and 
estimation of isophotal and rest-frame parameters, are described. In Section 4, we give the properties of the sample and main results of our study along with the comparison with earlier work. We discuss our results in Section 5 and give a summary and our conclusions in Section 6.

\section{THE OBSERVATIONAL DATA}

\subsection{Imaging Data}

The imaging data were originally obtained as part of a faint quasar survey using the Large Format Camera (LFC) on the $5 \mathrm{~m}$ Hale Telescope at Mount Palomar Observatory, with integrated exposures ranging from 1 to $4 \mathrm{hr}$ in $r, i$, and $z$ filters. The LFC is a mosaic of six $2048 \times 4096$ pixel CCDs, mounted at the prime focus of the Hale Telescope. The fields targeted were those of known SDSS high-redshift $(z>5)$ quasars in order to look for a local over-density of quasars (Mahabal et al. 2005). We obtained reduced images, which were bias subtracted, flat fielded, and processed to remove cosmic rays as a part of the original program. The deep LFC images have excellent signalto-noise ratio $(\mathrm{S} / \mathrm{N})$, permitting us to examine faint features, including the outermost extensions of the galaxies, down to $\sim 0.1 \%$ of the sky brightness.

In this paper we use a sample of galaxies selected from the LFC field SDSS 1208+0010, which spans an area of $13.56 \times$ $25^{\prime} .32$ centered on R.A. $=12^{\mathrm{h}} 08^{\mathrm{m}} 24^{\mathrm{s}}$ and decl. $=00^{\mathrm{d}} 10^{\mathrm{m}} 28^{\mathrm{s}}$ (J2000). The field was observed in the SDSS $i$ and $z$ filters with an integrated exposure of $2.51 \mathrm{hr}$ and $2.56 \mathrm{hr}$, respectively. The $i$-band image of the field is an average combination of 140 individual frames. The mean background count in the $i$-band image is 6278 ADU (analog-to-digital units), so with a gain of 1.1 , an accuracy of $\sim 0.1 \%$ is obtained in the estimation of the sky background.

All of the LFC fields are in the area of the sky covered by SDSS imaging. We therefore have imaging data in the SDSS $u$, $g$, and $r$ bands for our sample galaxies, in addition to the LFC $i$ - and $z$-band data, though not to the same depth. We have used the SDSS DR7 photometric catalog (Abazajian et al. 2009) to obtain the Petrosian half-light radii, apparent magnitudes, and Galactic extinction corrections at the position of each sample galaxy in all of the five bands.

\subsection{Sample Selection}

We used SExtractor (Bertin \& Arnouts 1996) to produce a catalog of all sources in the LFC field SDSS 1208+0010 and to identify galaxies from the catalog. SExtractor provides a stellarity index for star/galaxy separation of the detected objects: 0 for an object identified as a galaxy, 1 for a star, and some intermediate value for ambiguous objects, which are usually the fainter objects in the sample. For correct star/galaxy separation, and to have galaxies with sufficient brightness and size to ensure reliable morphological study, we select galaxies using objects from our SExtractor catalog which satisfy the following criteria: (1) SExtractor parameter value CLASS_STAR $<0.8$; (2) SDSS parameter value PhotoType $=$ GALAXY in the PhotoObjAll table of the SDSS DR7 photometric catalog (Abazajian et al. 2009); and (3) $m_{i}<20.5$, where $m_{i}$ is the $i$-band model magnitude of the object corrected for extinction, which is denoted by dered_ $i$ in the SDSS DR7 photometric catalog. To ensure that a sufficient number of pixels is available for surface photometry, we included in our sample only objects with the $i$-band SExtractor parameter ISOAREA_IMAGE $>165$. The area covered by the chosen pixels is $>21.74 \operatorname{arcsec}^{2}$ for every object in
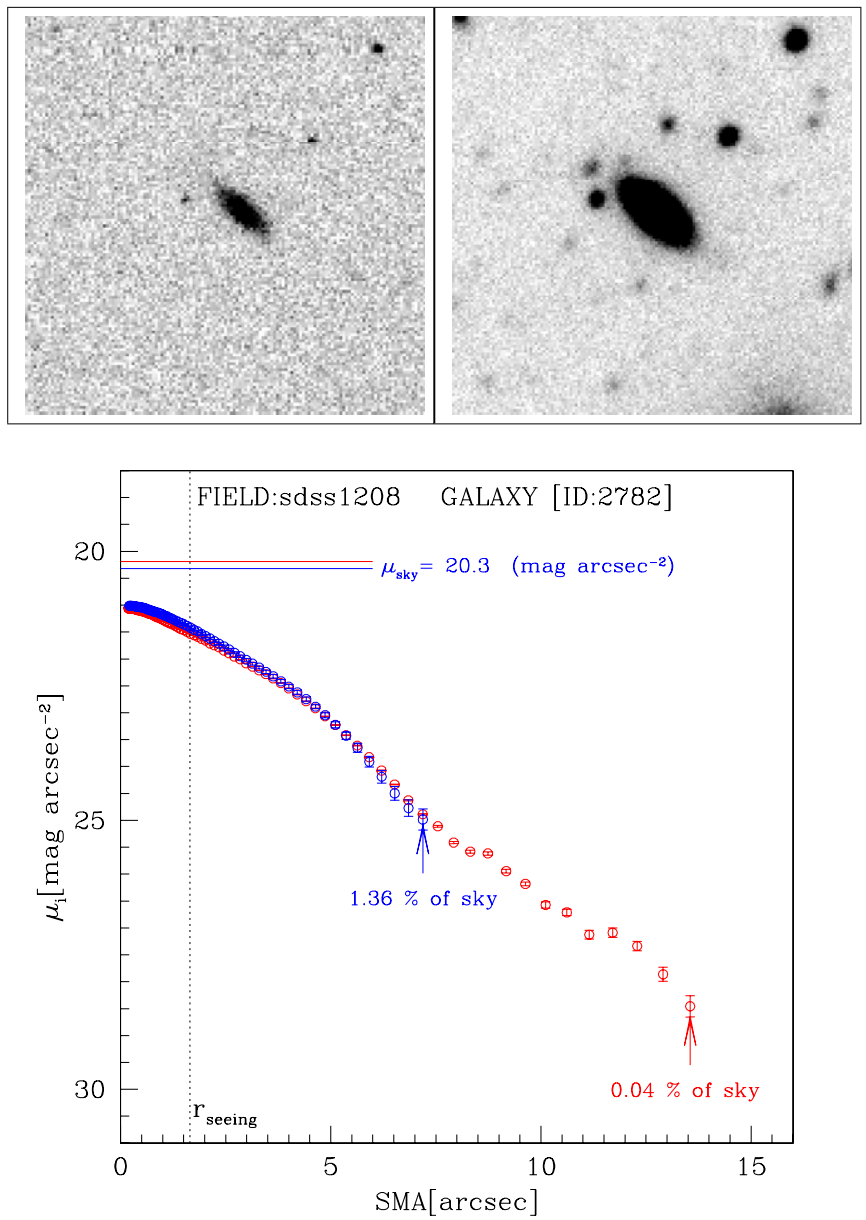

Figure 1. Surface brightness (SB) profiles of a galaxy from the sample, generated using SDSS (shown in blue) and LFC (shown in red) $i$-band images. The top panels show SDSS (left) and LFC (right) image cutouts $\left(\sim 55^{\prime \prime} \times 55^{\prime \prime}\right)$. The SB profiles are obtained by fitting ellipses up to a point along the semi-major axis, where the ratio of the mean isophotal intensity to the total error in the isophotal intensity drops to three in each image. The $1 \sigma$ error in the isophotal intensity is indicated for each point. The dotted vertical line shows the average seeing in the $i$-band LFC observation. The galaxy has $m_{i}=17.8$ and $z=0.1772$, as determined from AAT observation. The linear scale for the cosmology adopted (see Section 3) is $\sim 3 \mathrm{kpc} \operatorname{arcsec}^{-1}$.

(A color version of this figure is available in the online journal.)

the sample, with the intensity in every pixel being brighter than the analysis threshold of 26.0 mag $\operatorname{arcsec}^{-2}$, which corresponds to a $3 \sigma$ detection threshold. Through visual examination of the image, we have excluded objects which are near bright stars. Applying these criteria, we arrived at a sample of 266 galaxies.

Basic data for the target galaxies in field SDSS 1208+0010 are available in Table 1, which can be accessed online. Here, we give the first few entries from Table 1 as an illustration. The table gives an LFC serial number for each galaxy, from the SExtractor catalog generated by us, the SDSS ID, position, and photometric data from the DR7 photometric catalog (Abazajian et al. 2009), and finally the semi-major axis (in arcsec), $i$-band $\mathrm{SB}$ (in mag $\operatorname{arcsec}{ }^{-2}$ ), and $\mathrm{S} / \mathrm{N}$ of the outermost isophote to which an ellipse can be fitted (see Section 3 ).

In Figure 1, we show the $i$-band image of the largest galaxy from the LFC field, together with the SDSS $i$-band image of the same galaxy. The profiles of the SB along the semi-major axis of the two images are shown in the lower panels, up to a point where the ratio of the mean isophotal intensity to the total error in isophotal intensity is about three. These points 
Table 1

Basic Parameters of the Sample of Early-type Galaxies

\begin{tabular}{|c|c|c|c|c|c|c|c|c|c|c|c|c|}
\hline (1) & SDSS ID & R.A. & Decl. & $\begin{array}{c}u \\
(\mathrm{mag}) \\
(5)\end{array}$ & $\begin{array}{c}g \\
(\mathrm{mag}) \\
(6)\end{array}$ & $\begin{array}{c}r \\
(\mathrm{mag}) \\
(7)\end{array}$ & $\begin{array}{c}i \\
(\mathrm{mag}) \\
(8)\end{array}$ & $\begin{array}{c}z \\
(\mathrm{mag}) \\
(9)\end{array}$ & $\begin{array}{c}R_{50} \\
(\operatorname{arcsec}) \\
(10)\end{array}$ & $\begin{array}{c}\text { sma } \\
(\operatorname{arcsec}) \\
(11)\end{array}$ & $\begin{array}{c}\mu_{i} \\
\text { mag } \operatorname{arcsec}^{-2} \\
(12)\end{array}$ & $\mathrm{S} / \mathrm{N}$ \\
\hline LFC 462 & 588848899913548358 & 182.02545140 & -0.00014042 & $21.50 \pm 0.43$ & $21.06 \pm 0.13$ & $20.55 \pm 0.13$ & $20.57 \pm 0.21$ & $19.96 \pm 0.43$ & $1.07 \pm 0.16$ & 3.81 & $26.36 \pm 0.06$ & 17.25 \\
\hline LFC 525 & 587748929241612656 & 182.10068456 & 0.00579127 & $22.48 \pm 1.58$ & $20.76 \pm 0.09$ & $20.42 \pm 0.10$ & $20.13 \pm 0.12$ & $20.74 \pm 0.95$ & $1.10 \pm 0.11$ & 3.81 & $26.06 \pm 0.04$ & 26.07 \\
\hline LFC 554 & 587748929241547219 & 181.99182097 & 0.00889758 & $22.09 \pm 1.04$ & $21.89 \pm 0.24$ & $21.11 \pm 0.18$ & $19.90 \pm 0.09$ & $19.67 \pm 0.34$ & $1.28 \pm 0.07$ & 3.81 & $26.67 \pm 0.08$ & 12.50 \\
\hline LFC 558 & 587748929241547253 & 181.99959155 & 0.01032991 & $26.27 \pm 2.14$ & $21.73 \pm 0.24$ & $20.20 \pm 0.09$ & $19.44 \pm 0.07$ & $19.05 \pm 0.21$ & $1.01 \pm 0.07$ & 5.11 & $26.58 \pm 0.10$ & 10.06 \\
\hline LFC 622 & 587748929241546923 & 182.03581581 & 0.01761600 & $20.79 \pm 0.41$ & $19.26 \pm 0.03$ & $18.16 \pm 0.02$ & $17.70 \pm 0.02$ & $17.33 \pm 0.06$ & $1.38 \pm 0.02$ & 10.11 & $27.21 \pm 0.09$ & 11.88 \\
\hline LFC 633 & 587748929241547046 & 182.04195384 & 0.01802068 & $20.48 \pm 0.30$ & $20.09 \pm 0.06$ & $19.51 \pm 0.05$ & $19.11 \pm 0.05$ & $19.21 \pm 0.28$ & $1.26 \pm 0.05$ & 8.32 & $27.18 \pm 0.08$ & 13.77 \\
\hline LFC 663 & 587748929241612845 & 182.17111251 & 0.01778466 & $21.46 \pm 0.60$ & $21.97 \pm 0.26$ & $20.89 \pm 0.15$ & $20.06 \pm 0.10$ & $19.19 \pm 0.22$ & $1.14 \pm 0.08$ & 3.81 & $25.90 \pm 0.05$ & 23.48 \\
\hline LFC 677 & 587748929241547621 & 181.99102394 & 0.02035316 & $19.94 \pm 0.20$ & $22.14 \pm 0.42$ & $20.94 \pm 0.16$ & $20.98 \pm 0.33$ & $22.79 \pm 4.34$ & $0.80 \pm 0.17$ & 5.36 & $27.64 \pm 0.19$ & 5.11 \\
\hline LFC 725 & 587722983351583067 & 182.01578218 & 0.02274361 & $25.14 \pm 4.14$ & $21.58 \pm 0.27$ & $20.82 \pm 0.13$ & $20.75 \pm 0.30$ & $20.12 \pm 0.77$ & $1.32 \pm 0.37$ & 3.81 & $26.65 \pm 0.12$ & 8.92 \\
\hline LFC 741 & 587748929241547216 & 181.99078515 & 0.02434438 & $21.46 \pm 0.57$ & $21.65 \pm 0.19$ & $20.74 \pm 0.12$ & $20.19 \pm 0.12$ & $19.80 \pm 0.36$ & $0.97 \pm 0.09$ & 4.86 & $27.60 \pm 0.21$ & 4.73 \\
\hline LFC 824 & 587748929241547430 & 182.05600812 & 0.03170720 & $23.94 \pm 10.03$ & $23.14 \pm 2.52$ & $20.28 \pm 0.18$ & $20.44 \pm 0.65$ & $19.60 \pm 0.78$ & $0.74 \pm 0.33$ & 3.81 & $25.93 \pm 0.04$ & 28.48 \\
\hline LFC 848 & 587748929241547282 & 182.00945210 & 0.03522617 & $22.05 \pm 1.24$ & $20.93 \pm 0.14$ & $20.26 \pm 0.11$ & $20.13 \pm 0.17$ & $19.63 \pm 0.39$ & $1.00 \pm 0.12$ & 3.81 & $26.89 \pm 0.09$ & 11.38 \\
\hline LFC 925 & 587748929241612771 & 182.14531708 & 0.03984272 & $21.09 \pm 0.52$ & $21.00 \pm 0.13$ & $20.09 \pm 0.07$ & $19.76 \pm 0.09$ & $19.08 \pm 0.24$ & $1.27 \pm 0.07$ & 3.81 & $26.10 \pm 0.05$ & 21.72 \\
\hline LFC 954 & 587748929241612750 & 182.13801353 & 0.04149656 & $24.88 \pm 5.75$ & $23.04 \pm 0.87$ & $20.93 \pm 0.17$ & $20.11 \pm 0.14$ & $20.28 \pm 0.78$ & $1.06 \pm 0.15$ & 3.63 & $26.12 \pm 0.08$ & 12.67 \\
\hline LFC 985 & 587748929241547693 & 182.03813760 & 0.04718642 & $25.96 \pm 2.24$ & $25.08 \pm 1.99$ & $20.94 \pm 0.14$ & $19.70 \pm 0.08$ & $19.39 \pm 0.25$ & $1.21 \pm 0.10$ & 6.21 & $28.07 \pm 0.20$ & 4.93 \\
\hline
\end{tabular}

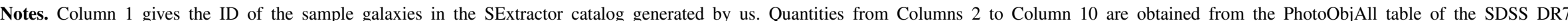

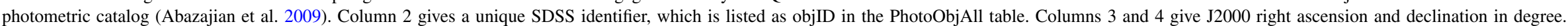

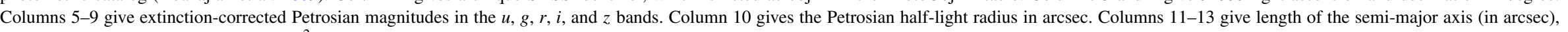
$i$-band surface brightness (in mag $\operatorname{arcsec}^{-2}$ ), and signal-to-noise ratio, respectively, of the outermost isophote in which an ellipse can be fitted (see Section 4).

(This table is available in its entirety in a machine-readable form in the online journal. A portion is shown here for guidance regarding its form and content.) 
are shown as arrows along the two profiles, with the SB level reached at that point given as a fraction of the sky brightness. Since the $\mathrm{S} / \mathrm{N}$ is the same for isophotes of the two images at the indicated points, it is clear that we reach $\sim 4$ mag arcsec $^{-2}$ fainter in SB in the LFC images compared with the SDSS images (see the Appendix). The mean value of the semi-major axis (sma) length for the outermost isophote for our sample galaxies is 5."41. The ellipse fitting process fails to converge before reaching the condition that isophotes have (mean isophotal intensity/total error in isophotal intensity) $\sim 3$ for most of the cases, principally because of crowding effects at the lowest brightness levels. For the sample, the mean $\mathrm{S} / \mathrm{N}$ reached at the outermost isophote is 14 . The average SB and its average error at the outermost isophote we could reach for our sample galaxies are $26.94 \mathrm{mag} \mathrm{arcsec}^{-2}$ and $0.12 \mathrm{mag} \mathrm{arcsec}^{-2}$, respectively.

\subsection{Bulge-Disk Decomposition}

We have performed bulge-disk decomposition and selected 132 galaxies with bulge-to-total luminosity ratio $B / T>0.4$ as being of early-type (see Rawat et al. 2007, for a discussion), which will form the basis for work reported in this paper. Here, the luminosities are estimated using parameters which define the large-scale structure of the galaxy. We assume that each galaxy consists of a bulge and a disk, and used the two-dimensional code GALFIT (Peng et al. 2002) to decompose the image of the galaxy into these components. The intensity profile of the bulge is modeled with the Sersic law (Sersic 1968):

$$
\Sigma(r)=\Sigma_{e} \exp \left[-2.303 b_{n}\left[\left(\frac{r}{r_{e}}\right)^{1 / n}-1\right]\right],
$$

where $r_{e}$ is the half-light radius, $\Sigma_{e}$ is the SB at $r_{e}$, and $n$ is the Sersic index. The disk is modeled with an exponential profile (Freeman 1970)),

$$
\Sigma(r)=\Sigma_{0} \exp \left[-\frac{r}{r_{d}}\right]
$$

where $\Sigma_{0}$ is the central $\mathrm{SB}$, and $r_{d}$ is the disk scale length. GALFIT obtains best-fit parameters by minimizing $\chi^{2}$ obtained by comparing the observed distribution of intensity in the twodimensional image of the galaxy with model images obtained using the radial profiles defined above and convolved with the point-spread function. After the decomposition, the total bulge and disk fluxes are obtained by integrating the best-fit profiles over all values of the semi-major axis.

\subsection{Spectroscopic Data}

The distances to most of our galaxies are unknown. However, we have obtained spectra for some of the galaxies in the LFC $1208+0010$ field using the 2dF/AAOmega multifiber system on the Anglo-Australian Telescope (AAT). For these galaxies, we can derive distances, absolute magnitudes, and linear sizes to facilitate comparison with other samples. These single-fiber spectra do not sample our galaxies consistently and were only intended for determining redshifts but they do confirm that most of our early-type galaxies selected on the basis of $B / T$ are indeed early-type galaxies.

We chose to observe the 266 selected galaxies from the field SDSS 1208+0010 (see Section 2.2) using the AAOmega multifiber spectrograph ${ }^{5}$ (Saunders et al. 2004) on the $4 \mathrm{~m} \mathrm{AAT.}$

\footnotetext{
5 See http://www.aao.gov.au/science/instruments/current/AAomega
}

AAOmega, which has 392 fibers each with $2^{\prime \prime}$ angular diameter, was used with gratings $385 R$ and $580 \mathrm{~V}$ in multi-object mode. The detectors used were English Electric Valve Co. (EEV) 4482 $2 \mathrm{k} \times 4 \mathrm{k}$ CCDs having pixel size $15 \mu \mathrm{m}$, allowing 10 pixels per fiber. The wavelength range covered by grating $580 \mathrm{~V}$ is $3700-5700 \AA$ with dispersion $1.0 \AA \mathrm{pixel}^{-1}$ and by grating $385 R$ is $5600-8800 \AA$ with dispersion $1.6 \AA \mathrm{pixel}^{-1}$. This setup of AAOmega provides a resolution of $R \sim 1300$.

The compactness of our field as compared with the $2^{\circ}$ field of view of AAOmega, together with a $30^{\prime \prime}$ restriction on the minimum separation between fibers, meant that it was not possible to observe all of the 266 galaxies simultaneously. We used the latest version of the AAOmega specific CONFIGURE program $^{6}$ for automatic fiber allocation. This program uses a "simulated annealing" algorithm (Miszalski et al. 2006) to maximize the number of objects observed. We assigned priorities to our targets as a function of fiber magnitude in the $g$ filter (fiberMag_g). We selected the positions of blank sky regions and suitable guide stars from the SDSS PhotoObjAll table, lying within the $2 \mathrm{dF}$ field but outside the central LFC field. SDSS images were visually inspected to ensure there was no contamination by other objects.

The publicly available $2 D F D R^{7}$ (Bailey et al. 2004) pipeline reduction system was used on each exposure to subtract bias and dark current and to carry out flat-fielding, sky-subtraction, and wavelength calibration. The blue- and red-arm spectra were spliced together after the spectra in each exposure set were co-added. Redshifts were determined using a version of the ZCODE software in manual mode (see Cannon et al. 2006, Sections 5.2 and 5.3). Each spectrum was visually inspected and assigned a redshift quality flag $Q$, having values from 0 to 6 based on (1) emission line/absorption line features identified in the spectrum and (2) cross-correlation value estimated by the software. The cross-correlation was done with that template spectrum which matched best with each observed spectrum. A larger value of $Q$ signifies a more reliable redshift measurement. Redshift measurements with quality $Q \geqslant 3$ were considered reliable, while $Q<3$ were assigned to unreliable redshifts or when no redshift was obtained. From experience with much larger samples of galaxies for other projects (e.g., Cannon et al. 2006), we believe that around $95 \%$ of redshifts flagged as reliable should be correct.

The median Petrosian half-light radius of our sample of galaxies is about $1^{\prime \prime}$.05, only slightly larger than the radius of the $2 \mathrm{dF} / \mathrm{AAOmega}$ fibers. Thus, for large nearby galaxies, our spectra sample only the nucleus, while for small distant galaxies, we integrate most of the light. The majority of our spectra were best fitted by an early-type galaxy template, confirming the validity of our selection criteria based on $B / T$. We defer further discussion of the spectra to a subsequent paper; here, we simply use the spectra to determine redshifts and hence distances.

AAOmega observations for galaxies in field SDSS 1208 were carried out in service mode with exposures of $1 \mathrm{hr}(3 \times 1200 \mathrm{~s})$ in 2006 June and for $1.5 \mathrm{hr}(3 \times 1800 \mathrm{~s})$ in $2007 \mathrm{March}$. In the observations of 2006 June, only 135 galaxies could be targeted. We attained a mean $\mathrm{S} / \mathrm{N}$ per pixel of $\sim 4$ in the blue and $\sim 10$ in the red spectra for targets with fiberMag $g \sim 21$. We obtained reliable redshifts for 55/135 (40\%) of the galaxies targeted and achieved $100 \%$ success for the brightest targets, i.e., for all 37 galaxies with fiberMag $g<21.2$. However, the success rate

\footnotetext{
6 See http://www.aao.gov.au/science/software/configure

7 http://www.aao.gov.au/science/software/2dfdr
} 


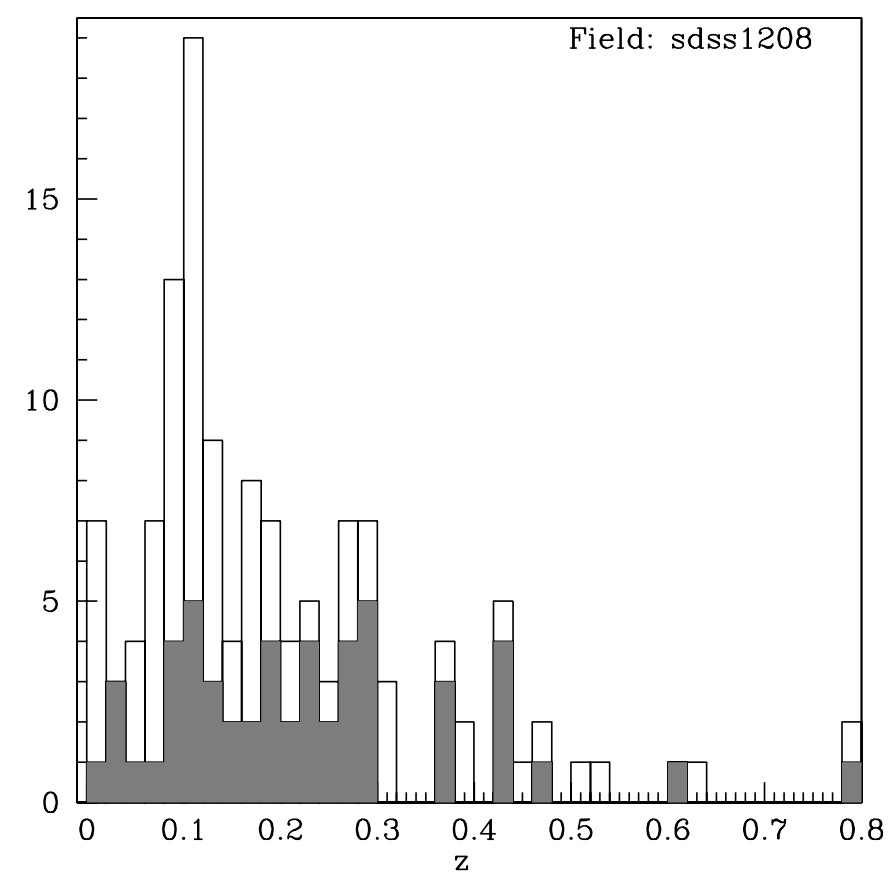

Figure 2. Redshift distribution of the 129 galaxies from field SDSS 1208. The shaded area shows the distribution of 53 early-type galaxies, which are part of this work.

fell rapidly for fainter galaxies. We observed the field SDSS 1208 again in 2007 March with increased exposure time and could target 121 galaxies from the field, including all those not targeted in 2006 plus 58 that failed to yield a redshift in the first run. By combining the spectra from the two observing runs we determined reliable redshifts for an additional 17 galaxies. It should be noted that our success in determining redshifts is higher when emission lines are present and that our fiber spectra sample only a $2^{\prime \prime}$ diameter patch of sky, which may contain a substantial fraction of the light from small distant galaxies but only the core of nearby galaxies. Thus, they do not give an unbiased sampling of the full set of galaxies or of their spectral types.

In Figure 2, we show the redshift distribution for all the galaxies in field SDSS 1208 for which we obtained redshifts. The redshifts of galaxies which we selected as early-type on the basis of $B / T$ are shown by the shaded area. It is evident that the proportion of early-type galaxies increases with redshift, with late-type galaxies being more common for $z<0.2$, while early-type galaxies are more common for $z>0.2$.

We have obtained the rest frame absolute magnitudes in the $u, g, r, i$, and $z$ bands for the galaxies for which we have redshift measurements using the equation

$$
M=m-\operatorname{DM}(z)-k(z)-A,
$$

where $z$ is the redshift; $m$ and $M$ are apparent and absolute magnitudes, respectively, in each band; and $k(z)$ and $A$ are the $k$-correction and Galactic extinction in that band. The distance modulus $\operatorname{DM}(z)$ is obtained using a flat $\lambda C D M$ cosmology with $\Omega_{m}=0.3, \Omega_{\Lambda}=0.7$, and $H_{0}=70 \mathrm{~km} \mathrm{~s}^{-1} \mathrm{Mpc}^{-1}$. The $k$-corrections have been obtained using the publicly available KCORRECT code (Blanton \& Roweis 2007), and the extinction values are obtained from the DR7 photometric catalog, which uses Galactic extinction maps published by Schlegel et al. (1998).

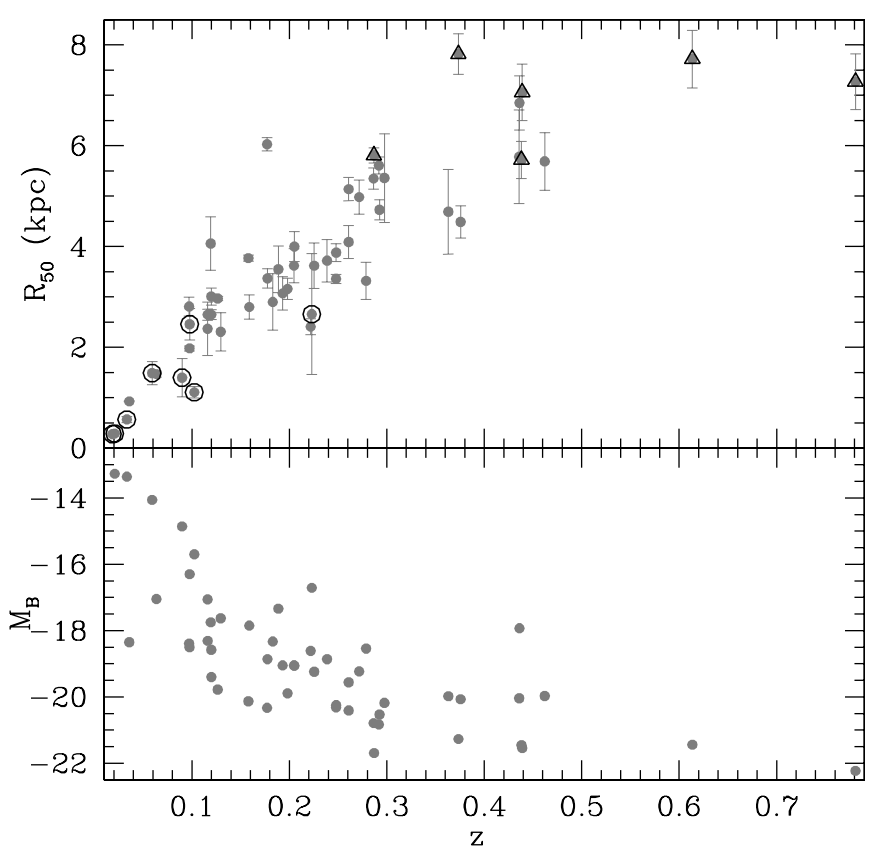

Figure 3. Lower panel shows a plot of redshift vs. $M_{\mathrm{B}}$ for the 53 galaxies of our sample; see text for details. The lowest redshift galaxies are mostly dwarfs, while those in the high-redshift tail are the brightest. The plot of redshift vs. Petrosian $R_{50}$ radius in the upper panel shows a good correlation. The dwarf galaxies (with $M_{\mathrm{B}}>-17$ ) are indicated by large circles, and the most luminous $\left(M_{\mathrm{B}}<-21\right)$ are indicated with triangle symbols.

To compare our results with earlier work, we have obtained Johnson $B$ and $V$ magnitudes using the transformations (Smith et al. 2002):

$$
\begin{gathered}
B=g+0.47(g-r)+0.17, \\
V=g-0.55(g-r)-0.03 .
\end{gathered}
$$

The apparent $B, V$ magnitudes are converted to absolute magnitudes using Equation (3) and $k$-corrections in the $B$ and $V$ bands are obtained from Poggianti (1997).

To summarize, from the two sets of AAT observations, we obtained reliable redshifts for 129 of the 266 galaxies in our original photometric sample; of these, 53 galaxies are included in the early-type sample discussed in this paper. The redshiftdependent parameters are listed in Table 2. In order to facilitate comparison with other samples of galaxies, plots of absolute $B$ magnitude and Petrosian $R_{50}$ radius against redshift are shown in Figure 3. The bulk of the galaxies have redshifts in the range $0.1<z<0.3$, with $M_{\mathrm{B}}$ between -17 and -21 and Petrosian $R_{50}$ from 2 to $6 \mathrm{kpc}$. There are half a dozen dwarfs with $z<0.1$ and a similar number of giants, mostly at high redshifts. The upper panel of Figure 3 shows a good correlation between Petrosian $R_{50}$ and $z$ for most of the galaxies.

\section{ISOPHOTAL ANALYSIS AND ESTIMATION OF PARAMETERS}

The early-type galaxies selected for our study are bulgedominated and therefore have isophotes which are close to elliptical in shape. It is our aim to measure morphological parameters, which describe the elliptical shape, as well as small deviations from it, as a function of galaxy properties. We performed ellipse fitting using the IRAF routine ellipse within STSDAS, which is based on a technique described by Jedrzejewski (1987). However, before the ellipse fitting, preprocessing using SExtractor has to be performed to obtain 
Table 2

Distance-dependent Parameters for 53 Galaxies with Redshifts

\begin{tabular}{|c|c|c|c|c|c|c|c|c|c|c|}
\hline (1) & (2) & $\begin{array}{c}R_{50} \\
(\mathrm{kpc}) \\
(3)\end{array}$ & $\begin{array}{c}s m a \\
(\mathrm{kpc}) \\
(4)\end{array}$ & $\begin{array}{c}M_{u} \\
(\mathrm{mag}) \\
(5)\end{array}$ & $\begin{array}{c}M_{g} \\
(\mathrm{mag}) \\
(6)\end{array}$ & $\begin{array}{c}M_{r} \\
(\mathrm{mag}) \\
(7)\end{array}$ & $\begin{array}{c}M_{i} \\
(\mathrm{mag}) \\
(8)\end{array}$ & $\begin{array}{c}M_{z} \\
(\mathrm{mag}) \\
(9)\end{array}$ & $\begin{array}{c}M_{\mathrm{B}} \\
(\mathrm{mag}) \\
(10)\end{array}$ & $\begin{array}{c}M_{\mathrm{V}} \\
(\mathrm{mag}) \\
(11)\end{array}$ \\
\hline LFC 558 & 0.4382 & $5.72 \pm 0.37$ & 28.97 & -18.48 & -21.90 & -22.49 & -22.85 & -23.07 & -21.45 & -22.26 \\
\hline LFC 622 & 0.1580 & $3.77 \pm 0.06$ & 27.59 & -19.24 & -20.66 & -21.43 & -21.78 & -22.11 & -20.13 & -21.12 \\
\hline LFC 633 & 0.1162 & $2.65 \pm 0.11$ & 17.50 & -18.26 & -18.73 & -19.27 & -19.54 & -19.47 & -18.31 & -19.06 \\
\hline LFC 663 & 0.6136 & $7.72 \pm 0.57$ & 25.75 & -21.39 & -22.18 & -23.40 & -23.79 & -24.52 & -21.44 & -22.88 \\
\hline LFC 824 & 0.2230 & $2.66 \pm 1.20$ & 13.68 & -17.42 & -17.94 & -20.20 & -19.88 & -20.67 & -16.71 & -19.21 \\
\hline LFC 848 & 0.2255 & $3.62 \pm 0.45$ & 13.80 & -18.46 & -19.60 & -20.01 & -20.02 & -20.48 & -19.24 & -19.86 \\
\hline LFC 985 & 0.4361 & $6.85 \pm 0.54$ & 35.11 & -19.53 & -19.47 & -22.37 & -23.17 & -23.21 & -17.93 & -21.10 \\
\hline LFC 988 & 0.2788 & $3.32 \pm 0.37$ & 16.13 & -17.20 & -19.09 & -19.92 & -20.69 & -21.08 & -18.54 & -19.58 \\
\hline LFC 1039 & 0.3632 & $4.69 \pm 0.84$ & 29.91 & -19.70 & -20.37 & -20.85 & -20.52 & -18.35 & -19.98 & -20.66 \\
\hline LFC 1056 & 0.0636 & $1.47 \pm 0.06$ & 9.70 & -16.05 & -17.47 & -18.02 & -18.18 & -18.19 & -17.05 & -17.81 \\
\hline LFC 1091 & 0.4359 & $5.78 \pm 0.93$ & 28.88 & -18.56 & -20.78 & -22.00 & -22.42 & -22.94 & -20.04 & -21.48 \\
\hline LFC 1411 & 0.1588 & $2.80 \pm 0.24$ & 10.45 & -17.72 & -18.35 & -19.06 & -19.16 & -19.15 & -17.85 & -18.77 \\
\hline LFC 1494 & 0.2919 & $5.61 \pm 0.17$ & 36.36 & -20.60 & -21.36 & -22.13 & -22.61 & -22.69 & -20.83 & -21.81 \\
\hline LFC 1504 & 0.2716 & $4.98 \pm 0.34$ & 27.07 & -18.55 & -19.75 & -20.50 & -21.05 & -20.73 & -19.23 & -20.19 \\
\hline LFC 1551 & 0.1829 & $2.90 \pm 0.56$ & 11.72 & -19.07 & -18.93 & -19.85 & -20.02 & -21.28 & -18.33 & -19.47 \\
\hline LFC 1613 & 0.1161 & $2.37 \pm 0.53$ & 11.27 & -17.10 & -17.47 & -17.99 & -17.76 & -17.65 & -17.06 & -17.79 \\
\hline LFC 1739 & 0.0332 & $0.57 \pm 0.06$ & 3.38 & -9.69 & -13.95 & -14.83 & -15.53 & -16.20 & -13.36 & -14.46 \\
\hline LFC 1772 & 0.1775 & $3.37 \pm 0.19$ & 16.90 & -16.78 & -19.34 & -20.00 & -20.00 & -20.20 & -18.86 & -19.73 \\
\hline LFC 1802 & 0.2925 & $4.73 \pm 0.20$ & 25.88 & -19.77 & -20.89 & -21.30 & -21.50 & -21.32 & -20.53 & -21.15 \\
\hline LFC 2159 & 0.2387 & $3.72 \pm 0.42$ & 14.40 & -18.33 & -19.53 & -20.60 & -20.95 & -22.08 & -18.86 & -20.15 \\
\hline LFC 2168 & 0.2480 & $3.88 \pm 0.18$ & 14.82 & -19.68 & -20.59 & -20.94 & -20.76 & -20.94 & -20.25 & -20.81 \\
\hline LFC 2177 & 0.2047 & $3.62 \pm 0.34$ & 17.16 & -18.26 & -19.50 & -20.12 & -19.98 & -20.39 & -19.05 & -19.87 \\
\hline LFC 2180 & 0.1024 & $1.11 \pm 0.11$ & 6.77 & -16.46 & -16.19 & -16.87 & -17.71 & -18.57 & -15.70 & -16.59 \\
\hline LFC 2181 & 0.2479 & $3.36 \pm 0.09$ & 19.84 & -19.46 & -20.74 & -21.28 & -21.46 & -21.78 & -20.32 & -21.07 \\
\hline LFC 2184 & 0.0357 & $0.93 \pm 0.01$ & 7.92 & -18.01 & -18.64 & -18.91 & -18.97 & -19.04 & -18.35 & -18.82 \\
\hline LFC 2310 & 0.1931 & $3.07 \pm 0.33$ & 12.24 & -19.22 & -19.47 & -20.02 & -20.10 & -20.61 & -19.05 & -19.80 \\
\hline LFC 2356 & 0.1193 & $4.06 \pm 0.53$ & 8.20 & -16.73 & -18.07 & -18.40 & -18.45 & -18.74 & -17.75 & -18.28 \\
\hline LFC 2362 & 0.0592 & $1.49 \pm 0.23$ & 7.84 & -15.51 & -14.99 & -16.61 & -17.28 & -17.74 & -14.06 & -15.91 \\
\hline LFC 2405 & 0.0899 & $1.40 \pm 0.38$ & 5.80 & -12.28 & -15.57 & -16.73 & -16.86 & -17.96 & -14.86 & -16.24 \\
\hline LFC 2414 & 0.2869 & $5.81 \pm 0.15$ & 37.72 & -21.21 & -22.22 & -22.97 & -23.28 & -23.56 & -21.69 & -22.66 \\
\hline LFC 2484 & 0.1887 & $3.55 \pm 0.46$ & 13.91 & -19.48 & -18.08 & -19.31 & -19.39 & -19.11 & -17.34 & -18.79 \\
\hline LFC 2525 & 0.2051 & $4.00 \pm 0.30$ & 12.83 & -18.26 & -19.44 & -19.90 & -20.07 & -20.00 & -19.06 & -19.72 \\
\hline LFC 2549 & 0.1264 & $2.97 \pm 0.04$ & 21.78 & -19.02 & -20.26 & -20.93 & -21.24 & -21.49 & -19.78 & -20.66 \\
\hline LFC 2692 & 0.1295 & $2.31 \pm 0.38$ & 11.79 & -14.39 & -17.83 & -17.90 & -17.70 & -19.00 & -17.63 & -17.90 \\
\hline LFC 2708 & 0.0184 & $0.28 \pm 0.02$ & 1.42 & -8.13 & -12.93 & -14.19 & -14.57 & -12.69 & -12.17 & -13.65 \\
\hline LFC 2712 & 0.1198 & $3.01 \pm 0.17$ & 16.30 & -17.70 & -19.21 & -20.21 & -20.74 & -21.25 & -18.58 & -19.79 \\
\hline LFC 2782 & 0.1772 & $6.03 \pm 0.13$ & 40.61 & -19.58 & -20.83 & -21.52 & -21.87 & -22.26 & -20.33 & -21.24 \\
\hline LFC 3072 & 0.4621 & $5.69 \pm 0.57$ & 29.86 & -20.85 & -20.65 & -21.73 & -21.94 & -22.48 & -19.97 & -21.28 \\
\hline LFC 3094 & 0.2864 & $5.35 \pm 0.21$ & 32.55 & -20.33 & -21.19 & -21.68 & -21.80 & -22.06 & -20.79 & -21.49 \\
\hline LFC 3218 & 0.2218 & $2.41 \pm 0.16$ & 16.56 & -18.37 & -19.02 & -19.53 & -19.74 & -19.92 & -18.61 & -19.33 \\
\hline LFC 3274 & 0.0977 & $2.46 \pm 0.31$ & 6.56 & -16.43 & -16.74 & -17.31 & -17.69 & -17.57 & -16.30 & -17.08 \\
\hline LFC 3292 & 0.4390 & $7.06 \pm 0.56$ & 35.24 & -20.69 & -21.76 & -21.85 & -22.06 & -21.94 & -21.54 & -21.84 \\
\hline LFC 3381 & 0.7809 & $7.27 \pm 0.55$ & 38.03 & -22.38 & -22.79 & -23.63 & -23.38 & -23.31 & -22.23 & -23.28 \\
\hline LFC 3430 & 0.1200 & $2.65 \pm 0.10$ & 24.12 & -18.87 & -19.89 & -20.58 & -20.91 & -21.26 & -19.40 & -20.30 \\
\hline LFC 3458 & 0.3757 & $4.49 \pm 0.32$ & 25.14 & -20.25 & -20.69 & -21.65 & -22.01 & -22.50 & -20.07 & -21.25 \\
\hline LFC 3508 & 0.2609 & $5.14 \pm 0.23$ & 35.25 & -19.75 & -20.97 & -21.82 & -22.27 & -22.49 & -20.41 & -21.47 \\
\hline LFC 4009 & 0.1981 & $3.16 \pm 0.21$ & 20.33 & -19.19 & -20.31 & -20.84 & -21.12 & -21.49 & -19.89 & -20.63 \\
\hline LFC 4059 & 0.3735 & $7.82 \pm 0.40$ & 52.06 & -19.50 & -21.67 & -22.17 & -22.43 & -22.36 & -21.27 & -21.98 \\
\hline LFC 4188 & 0.0975 & $1.98 \pm 0.05$ & 10.66 & -17.79 & -18.99 & -19.69 & -19.97 & -20.15 & -18.50 & -19.41 \\
\hline LFC 4248 & 0.0972 & $2.81 \pm 0.19$ & 14.96 & -17.78 & -18.73 & -19.10 & -19.24 & -19.55 & -18.39 & -18.96 \\
\hline LFC 4394 & 0.2608 & $4.09 \pm 0.33$ & 15.37 & -17.94 & -19.84 & -20.09 & -20.33 & -20.61 & -19.56 & -20.01 \\
\hline LFC 4536 & 0.2975 & $5.36 \pm 0.88$ & 16.88 & -19.52 & -20.46 & -20.68 & -21.02 & -21.17 & -20.18 & -20.61 \\
\hline LFC 4763 & 0.0208 & $0.29 \pm 0.01$ & 1.84 & -13.36 & -13.86 & -14.75 & -15.22 & -15.62 & -13.27 & -14.38 \\
\hline
\end{tabular}

Notes. Column 1 gives our SExtractor catalog ID. Column 2 gives spectroscopic redshift obtained by us. Columns 3 and 4 give Petrosian half-light radius and semi-major axis length in kiloparsec. Columns 5-11 give absolute magnitudes of galaxies in SDSS filters. Columns 10 and and 11 give Johnson $B$ and $V$ absolute magnitudes obtained using Equations (3) and (4).

a catalog of all detected objects in the field, a sky background image, and a segmentation image.

The segmentation image is a map of all detected objects in the field in which, for any given object, all of the pixels have a value equal to the running SExtractor catalog number corresponding to that object. The value of parameters used for estimation of the background in the SExtractor configuration file are BACK_SIZE 128; BACK_FILTERSIZE 3; BACKPHOTO_TYPE LOCAL; 
and BACKPHOTO_THICK 64 (see Bertin \& Arnouts 1996). The sky background image obtained is subtracted from the field image, and $150 \times 150$ pixel cutouts around the geometric center of our sample galaxies are made from the sky-subtracted image and the segmentation image. The cutouts of segmentation images are used to generate mask files for the surface photometry.

We use the geometric center, ellipticity, and position angle of sample galaxies, obtained from the SExtractor catalog, as initial values in the ellipse fitting. In the ellipse task, the image intensity is first sampled along a trial ellipse generated using these parameters, and the intensity string $I(\theta)$ is expanded in a Fourier series,

$$
I(\theta)=I_{0}+\sum_{n=1}^{N}\left[A_{n} \sin (n \theta)+B_{n} \cos (n \theta)\right],
$$

where $N$ is the highest harmonic fitted, and $\theta$ is the azimuthal angle measured from the major axis. ellipse uses the first- and second-order coefficients obtained for trial ellipses to iteratively improve the fitting. For perfectly elliptical isophotes, all of the Fourier coefficients except $I_{0}$, which is the mean isophotal intensity, should vanish at the end of the fitting process, and significant residuals can only be of order three or higher. We allowed the geometric center, ellipticity, and position angle to vary freely during fitting. Successive ellipses are fitted along the semi-major axis with a logarithmic step of 0.05 until the ratio is (mean isophotal intensity/total error in isophotal intensity) $\sim 3$, or the ellipse fitting process fails to converge. The output of ellipse is a table containing radial profiles of number of isophotal parameters along with uncertainties associated with them (see Busko 1996, for error estimation of isophotal parameters).

In their seminal papers on the isophotal shapes of elliptical galaxies, Bender et al. $(1988,1989)$ define structural parameters $a_{n} / a$ and $b_{n} / a$. The $A_{n}$ and $B_{n}$ provided by the ellipse table are normalized to the semi-major axis $a$ and the local intensity gradient intensity $(d I / d a)$ (ellipse output GRAD). The normalized $B_{n}$ coefficients can be converted to the $a_{n} / a$ parameter as

$$
\frac{a_{n}}{a}=B_{n} \sqrt{1-\epsilon}=B_{n} \sqrt{b / a}
$$

where $b$ is the semi-minor axis, and $\epsilon$ is the isophotal ellipticity (Milvang-Jensen \& Jørgensen 1999; Tremblay et al. 2007; Hao et al. 2006b). The factor of $\sqrt{1-\epsilon}=\sqrt{b / a}$ is needed to re-normalize the radial deviation to the equivalent radius $r=\sqrt{a b}$ (Milvang-Jensen \& Jørgensen 1999). The $a_{4} / a$ parameter, which is the most dominant coefficient for any isophote deviating from a pure ellipse, quantifies the deviation along the major axis of the isophote. Isophotes with $a_{4} / a<0$ have a "boxy" shape, while the shape of isophotes with $a_{4} / a>0$ is "disky" (Bender et al. 1988). The parameter $b_{n} / a$ can be related to the normalized $A_{n}$ parameter through a relation similar to Equation (6) but we do not use it in our present work.

The Fourier coefficients and morphological parameters vary along the semi-major axis, and we find that there are significant changes in values in the outer regions of the galaxies. It is therefore not enough to consider characteristic values of the parameters at a single fiducial distance from the center, as has been done by earlier works (see, e.g., Bender et al. 1988; Hao et al. 2006a), for investigating relationships between these parameters and other global galaxy properties. However, in comparing data for a large sample of galaxies, it is convenient to derive the mean values of isophote parameters in a few regions, rather than the full sets of isophotes. We therefore divide each galaxy into four regions defined by distance from the center along the semi-major axis: (1) seeing radius $r_{s}$ to $1.5 R_{50}$ (Region 1), (2) $1.5 R_{50}$ to $3.0 R_{50}$ (Region 2), (3) $3.0 R_{50}$ to $4.5 R_{50}$ (Region 3 ), and (4) semi-major axis $>4.5 R_{50}$ (Region 4 ), where $R_{50}$ is the Petrosian half-light radius. In each region, we obtain the mean value of various parameters weighted with the intensity (counts) and inversely weighted with the variance of the parameter. For example, the mean value of $a_{4} / a$ in Region 1 is obtained as

$$
\left\langle\frac{a_{4}}{a}\right\rangle=\frac{\int_{r_{s}}^{1.5 R_{50}} \frac{a_{4}}{a}(r) I(r)\left[\sigma_{\frac{a_{4}}{a}}(r)\right]^{-2} d r}{\int_{r_{s}}^{1.5 R_{50}} I(r)\left[\sigma_{\frac{a_{4}}{a}}(r)\right]^{-2} d r} .
$$

Note that not all galaxies have data in all four regions; for example, Region 1 does not exist if seeing radius $r_{s}>1.5 R_{50}$, while sometimes it is impossible to fit reliable ellipses in Regions 3 and 4 due to crowding and/or low S/N. Some of the derived parameters for our sample of galaxies are listed in Table 3 (only the first five galaxies are listed here as examples; the complete table can be accessed electronically).

Our method of obtaining characteristic parameter values is similar to the method used by Hao et al. (2006a) and Bender et al. (1988, 1989), except that (1) Hao et al. (2006a) considered a single characteristic parameter value in the region $2 r_{s}-1.5 R_{50}$, while Bender et al. $(1988,1989)$ defined the value in region $2 r_{s}-1.5 r_{e}$, where $r_{e}$ is the de Vaucouleur half-light radius, and (2) Bender et al. $(1988,1989)$ estimated the characteristic value of ellipticity as the maximum value in a peaked ellipticity profile, and as the value of the ellipticity at $1.5 r_{e}$ in case of a continuously increasing or decreasing ellipticity profile. We have chosen multiples of $R_{50}$ for defining the regions, as the value of $r_{e}$ is very sensitive to the goodness of fit and the range of radius chosen for fitting SB profiles with de Vaucouleurs law. The value of $R_{50}$ has been obtained from the SDSS DR7 galaxy catalog given by Blanton et al. (2005).

\section{OBSERVED PROPERTIES OF THE GALAXIES}

\subsection{Some Representative Galaxies}

In Figure 4, we show examples of three typical galaxies in our sample. The left panels show the observed isophotes (in blue) in each region of the galaxy along the semi-major axis, superimposed on gray-scale images from the LFC. The smooth red contours are the best-fitted ellipses to the observed contours (see Section 3). The right panels show the variation of $a_{4} / a$ along the semi-major axis of the galaxies.

These three examples represent common patterns for galaxies in our sample. The $a_{4} / a$ profile for the galaxy LFC1208_2549 shows diskiness (positive $a_{4} / a$ ) in all of the regions except in Region 2. The diskiness of galaxy LFC1208_2549 peaks in Region 3. The profile of LFC1208_1585 shows boxiness (negative $a_{4} / a$ ) in outer regions and small diskiness in the inner regions. The profile for the galaxy LFC1208_4681 remains constant along the semi-major axis with a value of $a_{4} / a$ close to zero, as the isophotes of this galaxy do not deviate much from the elliptical shape. The vertical lines in black color show the bins in which the mean values of isophotal parameters are computed. A red circle shows the mean value of $a_{4} / a$ in each bin. The red dotted vertical line in each plot marks the seeing 

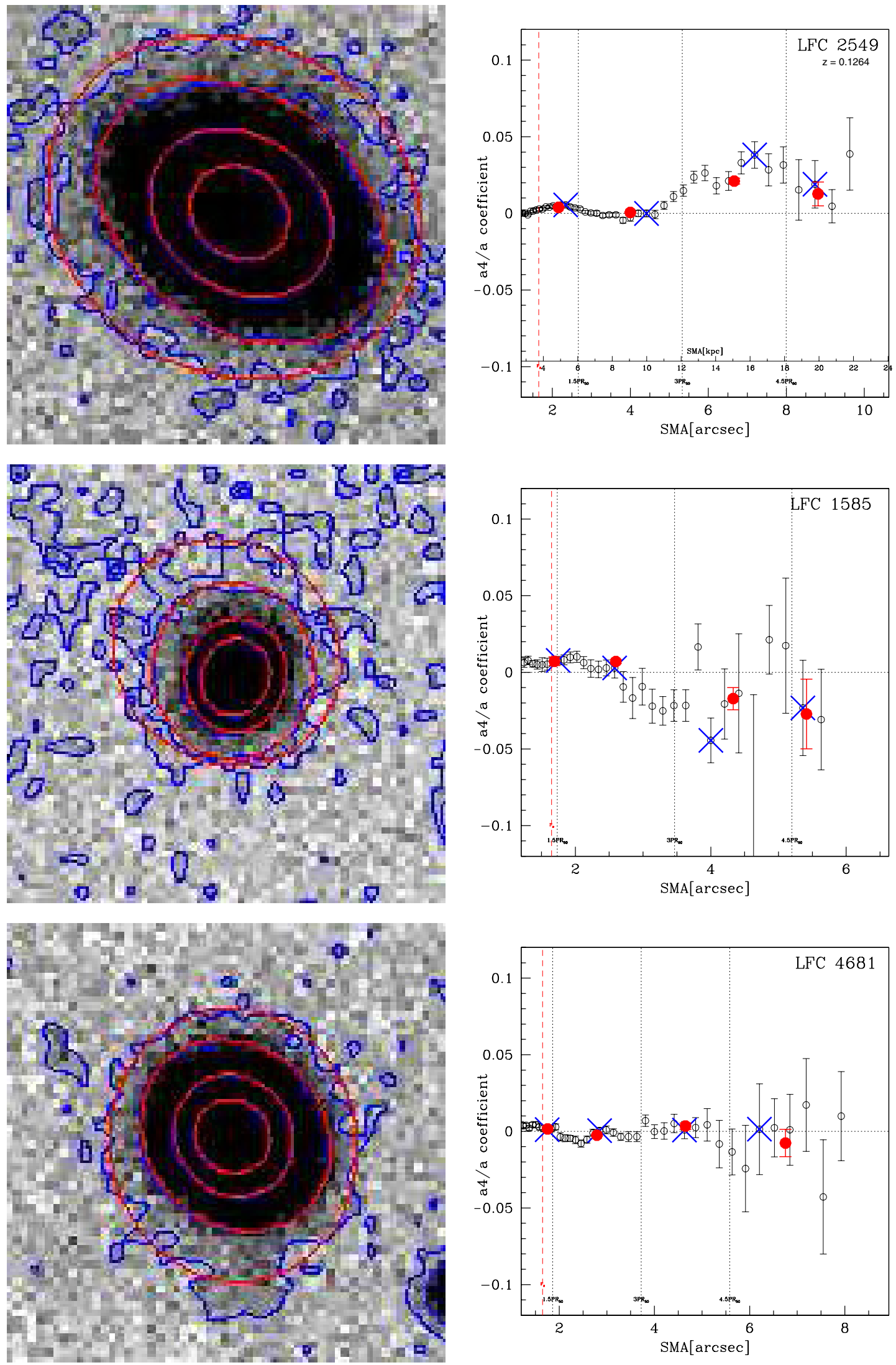

Figure 4. Left panels show $i$-band image cutouts $\left(20^{\prime \prime} \times 20^{\prime \prime}\right)$ for three of our galaxies. Isophotes (in blue) are shown together with the best-fitted ellipses (in red). The right panels show the variation of $a_{4} / a$ along the semi-major axis of each galaxy (with $1 \sigma$ error bars). The dotted vertical lines in black color indicate the bins of semi-major axis length in which the mean values of isophotal parameters are computed. A red circle shows the mean value of $a_{4} / a$ in each bin. The positions of the blue contours in each left-hand panel are marked by blue crosses in the corresponding right-hand panels. The region of each galaxy within the seeing radius (dashed vertical line) is excluded from the study. Most previous studies of isophotal parameters have been confined to only the innermost of our four bins.

(A color version of this figure is available in the online journal.) 
Table 3

Derived Parameters for a Sample of the LFC Galaxies

\begin{tabular}{|c|c|c|c|c|}
\hline $\begin{array}{l}\text { ID } \\
(1)\end{array}$ & $\begin{array}{l}B / T \\
(2)\end{array}$ & $\begin{array}{l}\left\langle\frac{a 3}{a}\right\rangle \\
(3)\end{array}$ & $\begin{array}{c}\left\langle\frac{a 4}{a}\right\rangle \\
(4)\end{array}$ & $\begin{array}{l}\langle\epsilon\rangle \\
(5)\end{array}$ \\
\hline LFC 462 & 0.89 & $\begin{aligned} \ldots & \\
3.52 \mathrm{e}-03 & \pm 2.25 \mathrm{e}-03 \\
7.01 \mathrm{e}-03 & \pm 4.73 \mathrm{e}-03\end{aligned}$ & $\begin{aligned} & \ldots \\
&-1.25 \mathrm{e}-03 \pm 2.16 \mathrm{e}-03 \\
& 6.91 \mathrm{e}-03 \pm 3.09 \mathrm{e}-03\end{aligned}$ & $\begin{array}{c}\ldots \\
0.057 \pm 0.0058 \\
0.048 \pm 0.0063\end{array}$ \\
\hline LFC 525 & 0.97 & $\begin{array}{c}\ldots \\
\ldots \\
5.52 \mathrm{e}-03 \pm 1.89 \mathrm{e}-03 \\
-6.61 \mathrm{e}-03 \pm 5.70 \mathrm{e}-03\end{array}$ & $\begin{array}{c}\cdots \\
\cdots \\
-1.09 \mathrm{e}-03 \pm 1.53 \mathrm{e}-03 \\
1.21 \mathrm{e}-02 \pm 5.41 \mathrm{e}-03\end{array}$ & $\begin{array}{c}\cdots \\
\cdots \\
0.070 \pm 0.0047 \\
0.023 \pm 0.0117\end{array}$ \\
\hline LFC 554 & 0.65 & $\begin{array}{l}-4.29 \mathrm{e}-03 \pm 1.96 \mathrm{e}-03 \\
-8.35 \mathrm{e}-03 \pm 3.77 \mathrm{e}-03\end{array}$ & $\begin{array}{l}1.00 \mathrm{e}-02 \pm 1.82 \mathrm{e}-03 \\
3.49 \mathrm{e}-03 \pm 3.75 \mathrm{e}-03\end{array}$ & $\begin{array}{l}0.094 \pm 0.0040 \\
0.115 \pm 0.0071\end{array}$ \\
\hline & & $\begin{array}{l}\ldots \\
\ldots\end{array}$ & $\begin{array}{l}\cdots \\
\ldots\end{array}$ & $\begin{array}{l}\cdots \\
\ldots\end{array}$ \\
\hline LFC 558 & 0.68 & $\begin{array}{r}6.77 \mathrm{e}-03 \pm 1.47 \mathrm{e}-03 \\
4.92 \mathrm{e}-03 \pm 1.60 \mathrm{e}-03 \\
-1.10 \mathrm{e}-02 \pm 1.19 \mathrm{e}-02\end{array}$ & $\begin{array}{r}1.88 \mathrm{e}-03 \pm 1.42 \mathrm{e}-03 \\
1.12 \mathrm{e}-04 \pm 1.56 \mathrm{e}-03 \\
-4.33 \mathrm{e}-03 \pm 1.28 \mathrm{e}-02\end{array}$ & $\begin{array}{l}0.038 \pm 0.0035 \\
0.081 \pm 0.0036 \\
0.164 \pm 0.0248\end{array}$ \\
\hline LFC 622 & 0.59 & $\begin{aligned}-4.88 \mathrm{e}-04 & \pm 1.06 \mathrm{e}-03 \\
-1.18 \mathrm{e}-03 & \pm 8.45 \mathrm{e}-04 \\
4.78 \mathrm{e}-02 & \pm 4.35 \mathrm{e}-03 \\
3.79 \mathrm{e}-02 & \pm 1.57 \mathrm{e}-02\end{aligned}$ & $\begin{array}{r}1.51 \mathrm{e}-02 \pm 4.42 \mathrm{e}-04 \\
1.29 \mathrm{e}-02 \pm 4.69 \mathrm{e}-04 \\
-1.47 \mathrm{e}-02 \pm 3.35 \mathrm{e}-03 \\
-2.56 \mathrm{e}-02 \pm 1.45 \mathrm{e}-02\end{array}$ & $\begin{array}{l}0.358 \pm 0.0018 \\
0.421 \pm 0.0013 \\
0.310 \pm 0.0075 \\
0.259 \pm 0.0257\end{array}$ \\
\hline LFC 633 & 0.66 & $\begin{array}{r}5.30 \mathrm{e}-03 \pm 7.48 \mathrm{e}-04 \\
1.59 \mathrm{e}-02 \pm 1.24 \mathrm{e}-03 \\
1.64 \mathrm{e}-02 \pm 4.29 \mathrm{e}-03 \\
-2.51 \mathrm{e}-01 \pm 7.91 \mathrm{e}-02\end{array}$ & $\begin{array}{l}-2.57 \mathrm{e}-03 \pm 6.74 \mathrm{e}-04 \\
-3.83 \mathrm{e}-03 \pm 1.11 \mathrm{e}-03 \\
-6.56 \mathrm{e}-04 \pm 4.22 \mathrm{e}-03 \\
-2.39 \mathrm{e}-02 \pm 2.74 \mathrm{e}-02\end{array}$ & $\begin{array}{l}0.213 \pm 0.0016 \\
0.219 \pm 0.0027 \\
0.269 \pm 0.0074 \\
0.102 \pm 0.0591\end{array}$ \\
\hline
\end{tabular}

Notes. Column 1 gives our SExtractor catalog ID. Column 2 gives the bulge-to-total luminosity ratio (see Section 2.3). Columns 3-5 give average isophotal parameters $a_{3} / a, a_{4} / a$, and ellipticity in four different regions for each galaxy. Average values of isophotal parameters in Regions 1-4 are listed, when available, in the first, second, third, and fourth rows, respectively, for each galaxy.

(This table is available in its entirety in a machine-readable form in the online journal. A portion is shown here for guidance regarding its form and content.)

radius of the image frame. The region of each galaxy within the seeing radius is excluded from the study. The necessity of considering radial variation of isophotal parameters, as we do in this paper, is clear from the profiles in the figure.

\subsection{Sample Properties}

In this section, we describe some of the basic properties of our sample and compare those with the properties of other samples used for similar studies. We look for evidence of systematic structural changes between the inner and outer regions of galaxies.

We first examine the differential probability distribution of ellipticity $\epsilon(=1-b / a)$, and shape parameters $a_{4} / a$ and $a_{3} / a$ in different regions of our sample galaxies.

We have fitted empirical Gaussian distribution functions (Equation (8)) to the histograms of various shape parameters for our galaxies, in different radial regions. Although the intrinsic distributions are unknown a priori, empirical fits to the data may be useful for comparison between data sets or with theoretical studies. The results of the fits are given in Table 4 and the fits along with the distribution of the above parameters are shown in Figure 5. In some cases, we could get better fits by fitting multiple Gaussians but the statistical significance and physical meaning of such fits was not clear. The differential probability distribution we use for $\epsilon, a_{4} / a$ and $a_{3} / a$ are of the form

$$
\begin{aligned}
& p(x) d x=\left[k_{1}+k_{2} x+k_{3} e^{-\frac{1}{2}\left(\frac{x-\mu_{1}}{\sigma_{1}}\right)^{2}}\right] d x, \\
& p(x) d x=\left[k_{1}+k_{2} x+k_{3} e^{-\frac{1}{2}\left(\frac{x-\mu_{1}}{\sigma_{1}}\right)^{2}}+k_{4} e^{-\frac{1}{2}\left(\frac{x-\mu_{2}}{\sigma_{2}}\right)^{2}}\right] d x .
\end{aligned}
$$

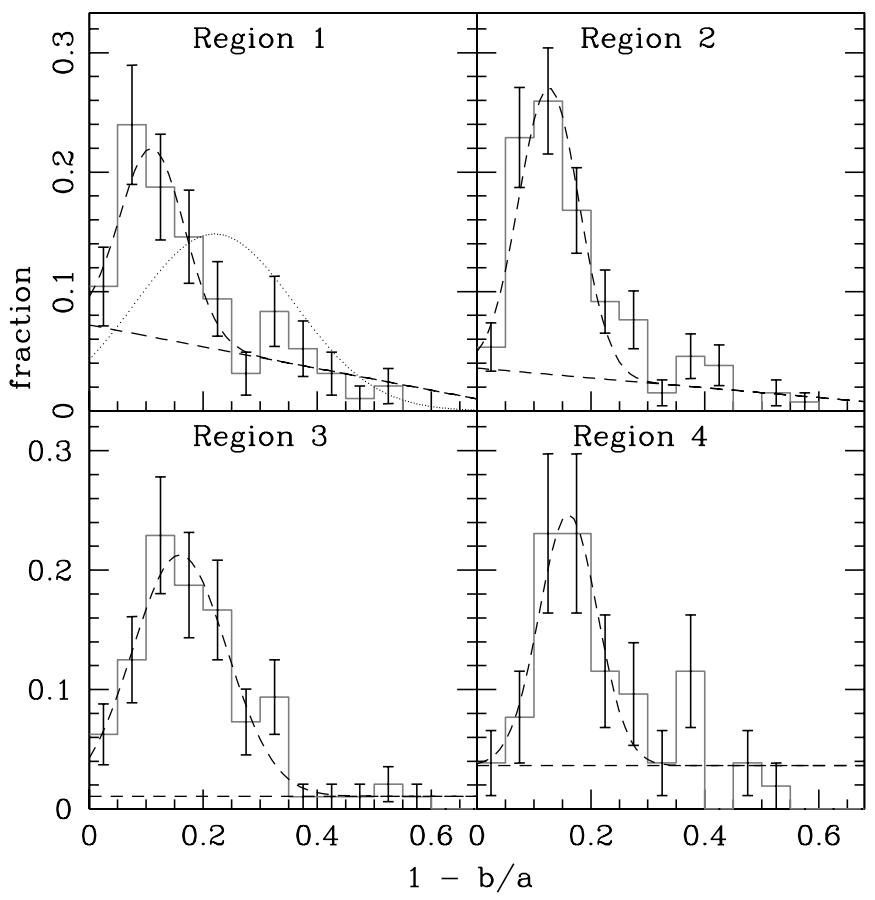

Figure 5. Distribution of ellipticity $\epsilon=(1-b / a)$, in different regions. The dashed lines are the best-fitted Gaussian distributions with baseline (see text and Table 4). The dotted curve in Region 1 is the best-fit Gaussian to the ellipticity distribution obtained by Hao et al. (2006a) for their sample. The error bars indicate $1 \sigma$ Poisson errors. 
Table 4

Values of Coefficients for Fitted Single and/or Double Gaussians (See Equation (8) and Figures 5-7)

\begin{tabular}{|c|c|c|c|c|c|c|c|c|c|c|}
\hline Parameter & $k_{1}$ & $k_{2}$ & $k_{3}$ & $\mu_{1}$ & $\sigma_{1}$ & $k_{4}$ & $\mu_{2}$ & $\sigma_{2}$ & $\chi^{2}$ & $\mathrm{rms}$ \\
\hline$\epsilon($ Region 1$)$ & $\begin{array}{r}0.072 \\
\pm 0.056\end{array}$ & $\begin{array}{l}-0.091 \\
\pm 0.131\end{array}$ & $\begin{array}{r}0.158 \\
\pm 0.049\end{array}$ & $\begin{array}{r}0.111 \\
\pm 0.012\end{array}$ & $\begin{array}{r}0.057 \\
\pm 0.037\end{array}$ & $\ldots$ & $\cdots$ & $\ldots$ & 1.05 & 0.025 \\
\hline$\epsilon($ Region 2$)$ & $\begin{array}{r}0.036 \\
\pm 0.045 \\
\end{array}$ & $\begin{array}{l}-0.041 \\
\pm 0.094\end{array}$ & $\begin{array}{r}0.240 \\
\pm 0.040 \\
\end{array}$ & $\begin{array}{r}0.127 \\
\pm 0.009 \\
\end{array}$ & $\begin{array}{r}0.053 \\
\pm 0.013 \\
\end{array}$ & $\cdots$ & $\cdots$ & $\ldots$ & 1.45 & 0.023 \\
\hline$\epsilon($ Region 3$)$ & $\begin{array}{r}0.011 \\
\pm 0.014 \\
\end{array}$ & $\begin{array}{r}0.0 \\
\pm 0.0 \\
\end{array}$ & $\begin{array}{r}0.202 \\
\pm 0.017 \\
\end{array}$ & $\begin{array}{r}0.159 \\
\pm 0.008 \\
\end{array}$ & $\begin{array}{r}0.083 \\
\pm 0.012 \\
\end{array}$ & $\ldots$ & $\cdots$ & $\cdots$ & 1.03 & 0.022 \\
\hline$\epsilon($ Region 4$)$ & $\begin{array}{r}0.036 \\
\pm 0.018 \\
\end{array}$ & $\begin{array}{r}0.0 \\
\pm 0.0 \\
\end{array}$ & $\begin{array}{r}0.210 \\
\pm 0.032 \\
\end{array}$ & $\begin{array}{r}0.160 \\
\pm 0.009 \\
\end{array}$ & $\begin{array}{r}0.051 \\
\pm 0.020 \\
\end{array}$ & $\ldots$ & $\ldots$ & $\ldots$ & 1.01 & 0.033 \\
\hline$\frac{a_{4}}{a}($ Region 1$)$ & $\begin{array}{r}0.0 \\
\pm 0.0 \\
\end{array}$ & $\begin{array}{r}0.0 \\
\pm 0.0 \\
\end{array}$ & $\begin{array}{r}0.0486 \\
\pm 0.0152 \\
\end{array}$ & $\begin{array}{r}0.0010 \\
\pm 0.0098 \\
\end{array}$ & $\begin{array}{r}0.0131 \\
\pm 0.0053 \\
\end{array}$ & $\begin{array}{r}0.3143 \\
\pm 0.0205 \\
\end{array}$ & $\begin{array}{r}0.0022 \\
\pm 0.0001 \\
\end{array}$ & $\begin{array}{r}0.0042 \\
\pm 0.0005 \\
\end{array}$ & 1.04 & 0.010 \\
\hline$\frac{a_{4}}{a}$ (Region 2$)$ & $\begin{array}{r}0.0064 \\
\pm 0.3322 \\
\end{array}$ & $\begin{array}{l}-0.0726 \\
\pm 2.1186 \\
\end{array}$ & $\begin{array}{c}0.5163 \\
\pm 0.09308 \\
\end{array}$ & $\begin{array}{r}0.0003 \\
\pm 0.0007 \\
\end{array}$ & $\begin{array}{r}0.0016 \\
\pm 0.0024 \\
\end{array}$ & $\begin{array}{r}0.1256 \\
\pm 0.5300 \\
\end{array}$ & $\begin{array}{r}0.0015 \\
\pm 0.2297 \\
\end{array}$ & $\begin{array}{r}0.0093 \\
\pm 0.2068 \\
\end{array}$ & 1.14 & 0.013 \\
\hline$\frac{a_{4}}{a}$ (Region 3$)$ & $\begin{array}{r}0.0149 \\
\pm 0.0040 \\
\end{array}$ & $\begin{array}{l}-0.0273 \\
\pm 0.0300 \\
\end{array}$ & $\begin{array}{r}0.0683 \\
\pm 0.0632 \\
\end{array}$ & $\begin{array}{l}-0.0270 \\
\pm 0.0038 \\
\end{array}$ & $\begin{array}{r}0.0011 \\
\pm 0.0002 \\
\end{array}$ & $\begin{array}{r}0.1240 \\
\pm 0.0107 \\
\end{array}$ & $\begin{array}{r}0.00001 \\
\pm 0.00002 \\
\end{array}$ & $\begin{array}{r}0.0079 \\
\pm 0.0013 \\
\end{array}$ & 0.92 & 0.020 \\
\hline$\frac{a_{4}}{a}$ (Region 4$)$ & $\begin{array}{r}0.0268 \\
\pm 0.0076 \\
\end{array}$ & $\begin{array}{l}-0.0267 \\
\pm 0.1084\end{array}$ & $\begin{array}{r}0.1141 \\
\pm 0.0810\end{array}$ & $\begin{array}{l}-0.0256 \\
\pm 0.0321 \\
\end{array}$ & $\begin{array}{r}0.0014 \\
\pm 0.0005\end{array}$ & $\begin{array}{r}0.1407 \\
\pm 2.6219\end{array}$ & $\begin{array}{r}0.0110 \\
\pm 0.0004 \\
\end{array}$ & $\begin{array}{r}0.0030 \\
\pm 0.0010\end{array}$ & 0.53 & 0.021 \\
\hline$\frac{a_{3}}{a}($ Region 1$)$ & $\begin{array}{r}0.0 \\
\pm 0.0 \\
\end{array}$ & $\begin{array}{r}0.0 \\
\pm 0.0 \\
\end{array}$ & $\begin{array}{r}0.4510 \\
\pm 0.0153 \\
\end{array}$ & $\begin{array}{l}-0.0009 \\
\pm 0.0003\end{array}$ & $\begin{array}{r}0.0078 \\
\pm 0.0004 \\
\end{array}$ & $\begin{array}{r}0.0135 \\
\pm 0.0068 \\
\end{array}$ & $\begin{array}{l}-0.0112 \\
\pm 0.0234\end{array}$ & $\begin{array}{r}0.0712 \\
\pm 0.0208 \\
\end{array}$ & 0.92 & 0.008 \\
\hline$\frac{a_{3}}{a}$ (Region 2) & $\begin{array}{r}0.0 \\
\pm 0.0\end{array}$ & $\begin{array}{r}0.0 \\
\pm 0.0 \\
\end{array}$ & $\begin{array}{r}0.3639 \\
\pm 0.0307\end{array}$ & $\begin{array}{l}-0.0011 \\
\pm 0.0010\end{array}$ & $\begin{array}{r}0.0105 \\
\pm 0.0009\end{array}$ & $\ldots$ & $\ldots$ & $\ldots$ & 1.38 & 0.023 \\
\hline$\frac{a_{3}}{a}$ (Region 3$)$ & $\begin{array}{r}0.0 \\
\pm 0.0 \\
\end{array}$ & $\begin{array}{r}0.0 \\
\pm 0.0 \\
\end{array}$ & $\begin{array}{r}0.2253 \\
\pm 0.0112 \\
\end{array}$ & $\begin{array}{l}-0.0024 \\
\pm 0.0008 \\
\end{array}$ & $\begin{array}{r}0.0142 \\
\pm 0.0009 \\
\end{array}$ & $\begin{array}{r}0.0593 \\
\pm 0.0322 \\
\end{array}$ & $\begin{array}{r}0.0513 \\
\pm 0.0043 \\
\end{array}$ & $\begin{array}{r}0.0140 \\
\pm 0.0056 \\
\end{array}$ & 1.01 & 0.012 \\
\hline$\frac{a_{3}}{a}$ (Region 4$)$ & $\begin{array}{r}0.0 \\
\pm 0.0\end{array}$ & $\begin{array}{r}0.0 \\
\pm 0.0\end{array}$ & $\begin{array}{r}0.1081 \\
\pm 0.0131\end{array}$ & $\begin{array}{r}0.0046 \\
\pm 0.0061\end{array}$ & $\begin{array}{r}0.0352 \\
\pm 0.0062\end{array}$ & $\begin{array}{r}0.0090 \\
\pm 0.0231\end{array}$ & $\begin{array}{r}0.0291 \\
\pm 0.0302\end{array}$ & $\begin{array}{r}0.0036 \\
\pm 0.0009\end{array}$ & 0.93 & 0.023 \\
\hline
\end{tabular}

The coefficients $k_{3}, k_{4}, \mu_{1}, \mu_{2}, \sigma_{1}$, and $\sigma_{2}$ are the amplitude, mean, and the standard deviation of the fitted Gaussian. The coefficients $k_{1}$ and $k_{2}$ are zero point and slope of the baseline for the Gaussian. The results of the fit are given in Table 4 and the fits along with the distribution of the above parameters are shown in Figures 5-7.

The ellipticity distribution of the sample used by Hao et al. (2006a) (dotted curve in Region 1, upper left panel of Figure 5) shows a peak around $\epsilon \sim 0.2$. The main peak in our sample, at $\epsilon \sim 0.1$, indicates the presence of a larger fraction of rounder galaxies. Both distributions of ellipticity drop to zero for $\epsilon>0.5$. This difference in the ellipticity distributions is presumably because of the very different data sets. The galaxies of Hao et al. (2006a) are all large and relatively nearby, with $z<0.05$, selected over a large area of the sky. Most of our galaxies are much smaller and more distant, with a redshift distribution peaking at $z \sim 0.1$ and extending to beyond 0.5 (see Figure 2).

If Gaussian functions are fitted to the ellipticity distributions in Figure 5, we find that as we go from Region 2 to Region 4, the peak of the distribution occurs at around $0.13,0.16$, and 0.16 , i.e., the peak shifts slightly toward more flattened ellipses. Overall, the distributions of ellipticity in Regions 1 and 2 are similar to each other, as are those for Regions 3 and 4, but there are significant differences between the inner and outer regions.

Figure 6 shows the distribution of the quadrupole parameter $a_{4} / a$ for our sample. The distribution in Region 1 shows a slight excess of disky isophotes (positive $a_{4} / a$ values), although not as strong as that found at similar radii by Hao et al. (2006a) and shown by the blue curve. However, the distribution we find in Region 2 (upper right panel) does resemble that of Hao et al. (2006a). As in the case of the ellipticity plots in Figure 5, the

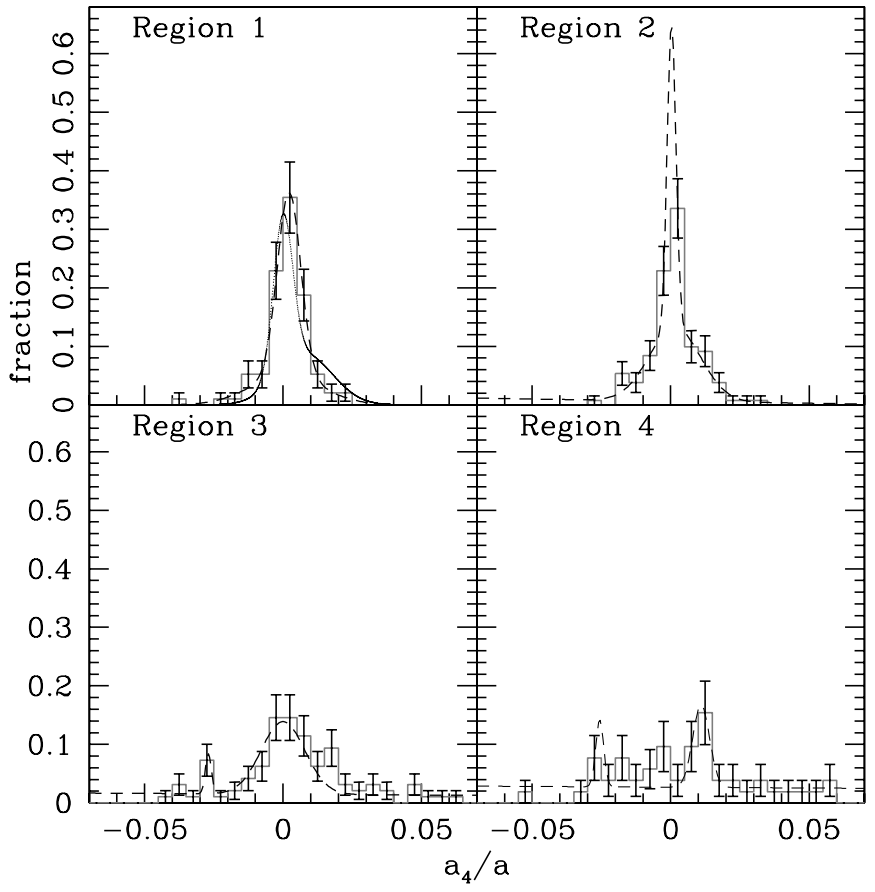

Figure 6. Distribution of $a_{4} / a$ parameters in different regions. The dashed lines are the best-fitted single or double Gaussians, (see text and Table 3). The dotted curve in Region 1 is the best-fit Gaussian to the $a_{4} / a$ distribution obtained by Hao et al. (2006a) for their sample. The error bars indicate $1 \sigma$ Poisson errors.

histograms for $a_{4} / a$ in Regions 1 and 2 are similar to each other but quite different from those for Regions 3 and 4.

The $a_{3} / a$ parameter quantifies the deviation from pure ellipse that occur along the observed isophote every $120^{\circ}$. Pasquali et al. 
Table 5

Results of a Two Sample K-S Test for the $a_{4} / a$ Parameter

\begin{tabular}{|c|c|c|c|c|c|c|c|c|}
\hline \multirow[t]{2}{*}{ Sample } & \multicolumn{2}{|c|}{ Region 1} & \multicolumn{2}{|c|}{ Region 2} & \multicolumn{2}{|c|}{ Region 3} & \multicolumn{2}{|c|}{ Region 4} \\
\hline & $D^{\mathrm{a}}$ & $P^{\mathrm{b}}$ & $D$ & $P$ & $D$ & $P$ & $D$ & $P$ \\
\hline Hao et al. (2006a) & 0.15 & 0.041 & 0.13 & 0.038 & 0.22 & $5.97 \mathrm{e}-04$ & 0.29 & $5.81 \mathrm{e}-04$ \\
\hline Region 1 & $\ldots$ & $\ldots$ & 0.08 & 0.855 & 0.25 & 0.005 & 0.35 & $6.41 \mathrm{e}-04$ \\
\hline Region 2 & $\ldots$ & $\ldots$ & $\ldots$ & $\ldots$ & 0.21 & 0.014 & 0.33 & $5.44 \mathrm{e}-04$ \\
\hline Region 3 & $\ldots$ & $\ldots$ & $\ldots$ & $\ldots$ & $\ldots$ & $\ldots$ & 0.16 & 0.383 \\
\hline
\end{tabular}

Notes.

a The K-S statistic, $D$, is defined as the maximum value of the absolute difference between two cumulative distribution functions, where the cumulative distribution function is obtained from the list of data points of each sample on which the $\mathrm{K}-\mathrm{S}$ test is applied.

b $P$ gives the level of significance with which the null hypothesis may be accepted. Small values of $P$ imply that the cumulative distribution function of two samples tested are significantly different (see Press et al. 1992). The confidence that both the population do not belong to the same parent distribution is given by $(1-P) \times 100$.

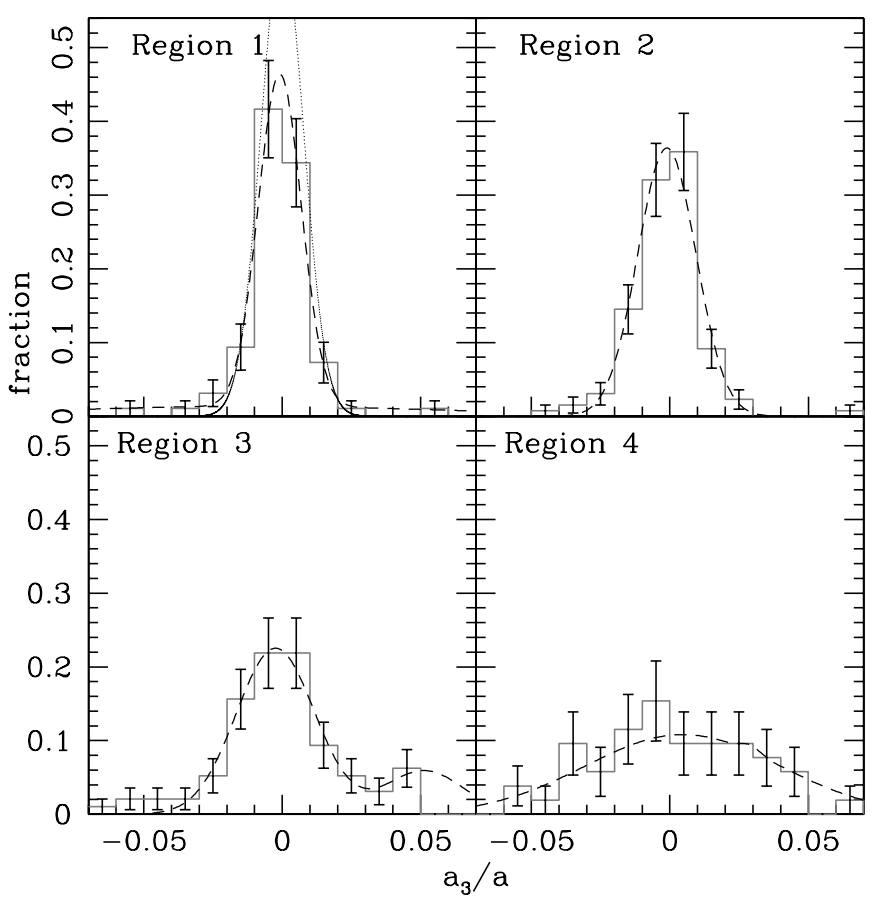

Figure 7. Distribution of $a_{3} / a$ parameters in different regions. The dashed lines are the best-fitted single or double Gaussians (see text and Table 4). The dotted curve in Region 1 is the best-fit Gaussian to the $a_{3} / a$ distribution obtained by Hao et al. (2006a) for their sample. The error bars indicate $1 \sigma$ Poisson errors.

(2007) have suggested that such deviations may be attributed to the presence of dust or clumps present within the galaxy. But the distribution of the dust can be irregular in the galaxy and can also give rise to higher order Fourier coefficients. It may be better to interpret $a_{3} / a$ as a structural parameter, possibly indicating dynamical effects from galaxy interactions or mergers. Figure 7 shows the distribution of this parameter for our sample.

The distribution of $a_{3} / a$ in Region 1 is reasonably fitted by a sum of two Gaussian functions with mean -0.0009 and -0.0112 with dispersions 0.0078 and 0.0712 . The value of $\chi^{2}$ per degree of freedom for the fit is $\chi_{v}^{2}=0.92$. In comparison, the distribution of the Hao et al. (2006a) sample is reasonably fitted by a single Gaussian with zero mean and dispersion of 0.0032. In our sample, the peaks of distribution in Region 1 and Region 2 occur at negative values of the parameter $a_{3} / a$, while one peak of the double Gaussian in Region 3 and both peaks of the double Gaussian in Region 4 occur at positive values of parameter $a_{3} / a$ (see Table 4 ). It is not known as to which property of galaxy determines the sign of the $a_{3} / a$ parameter. The findings of Jog \& Maybhate (2006) suggest that non-zero values of the $a_{3} / a$ parameter along with the presence of lopsidedness in the inner regions of galaxies can be interpreted as a signature of dynamically unrelaxed behavior in the inner regions of the galaxies. These authors have Fourieranalyzed the central few kpc of advanced mergers of galaxies using images from the Two Micron All Sky Survey and have obtained amplitudes and phases of Fourier components $m=1$, 2,3 , and 4. Their analysis indicates that in the case of mergers, the amplitudes $A 1$ and $A 2$ (for $m=1$ and 2, respectively) dominate over $m=3$ and 4 , and $A 3$ is important only when $A 1$ is large. A1 denotes the amplitude for the lopsidedness and is an indicator of mass asymmetry measured with respect to the constant center (see Section 3.2 of their paper for details). The study of lopsidedness in our sample galaxies should reveal whether the peaks of the $a_{3} / a$ distribution around non-zero values in the inner regions of our galaxies can be interpreted as a signature of dynamically unrelaxed inner regions. Though we do not understand the reason for the occurrence of peaks at positive values of $a_{3} / a$ in Region 3 and in Region 4, we can say that the processes which affect isophotal shape are different in the inner and outer regions.

We next look for correlations between the values for various shape parameters for individual galaxies, when adjacent radial regions are compared.

Figure 8 shows that there is a strong correlation between the ellipticity parameters in the innermost Regions 1 and 2 (upper panel). However, there is a significant change going from Region 2 to Region 3 (lower left); while most of the higher ellipticity galaxies remain correlated (those with $\epsilon>0.3$ in Regions 1 and 2), about half of the nearly circular galaxies become more flattened. The outer most parts (Regions 3 and 4, lower right panel) show more scatter, and fewer galaxies have data in Region 4 but an overall correlation remains.

We show similar diagrams for the shape parameter $a_{4} / a$ in Figure 9. The dominant feature is a large increase in the scatter of points between Regions 2 and 3, shown in the lower right panel. The other two plots, for the outer Regions 3 and 4 (upper panel) and the inner Regions 1 and 2 (lower left), show some correlation between adjacent regions, with few points populating the top left and bottom right quadrants. 

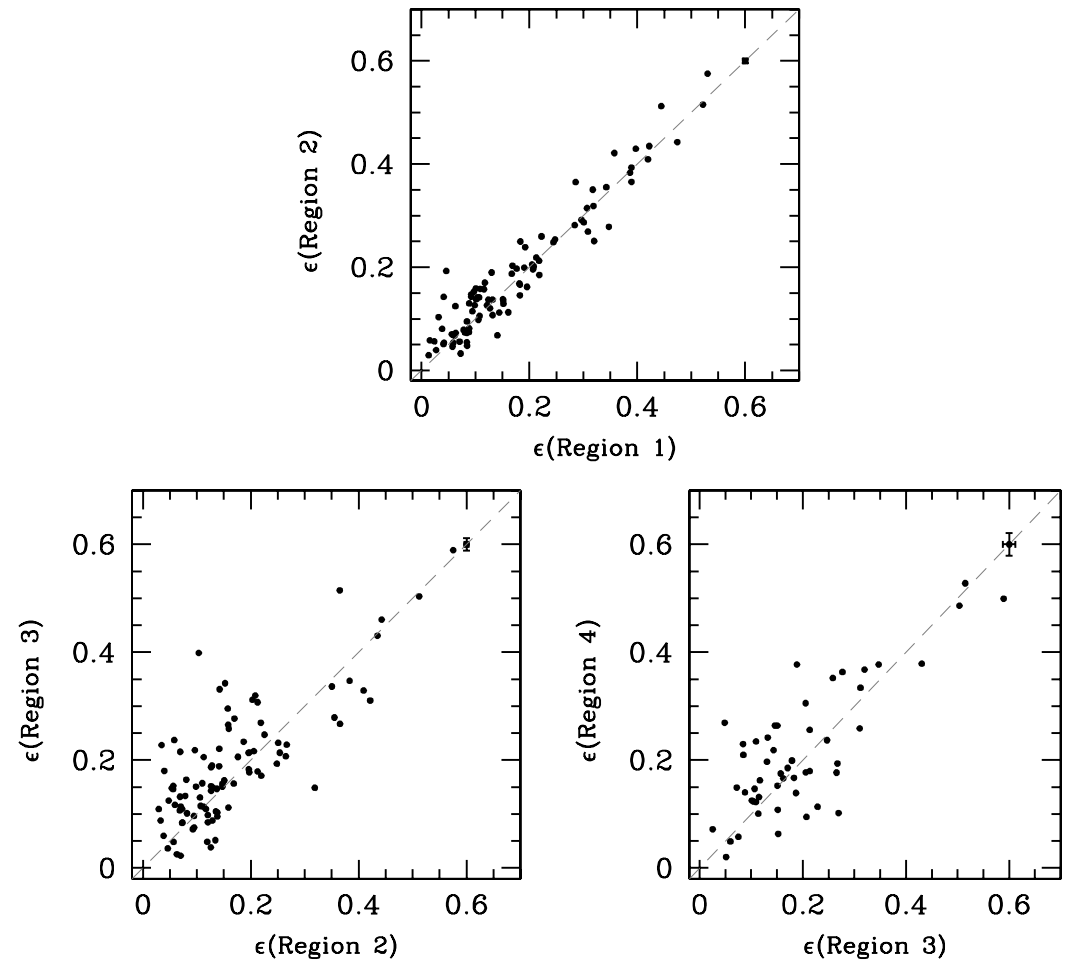

Figure 8. Correlation of ellipticity $(\epsilon=1-b / a)$ parameters from neighboring regions. The median error bars are shown at the top right-hand corner in each panel.
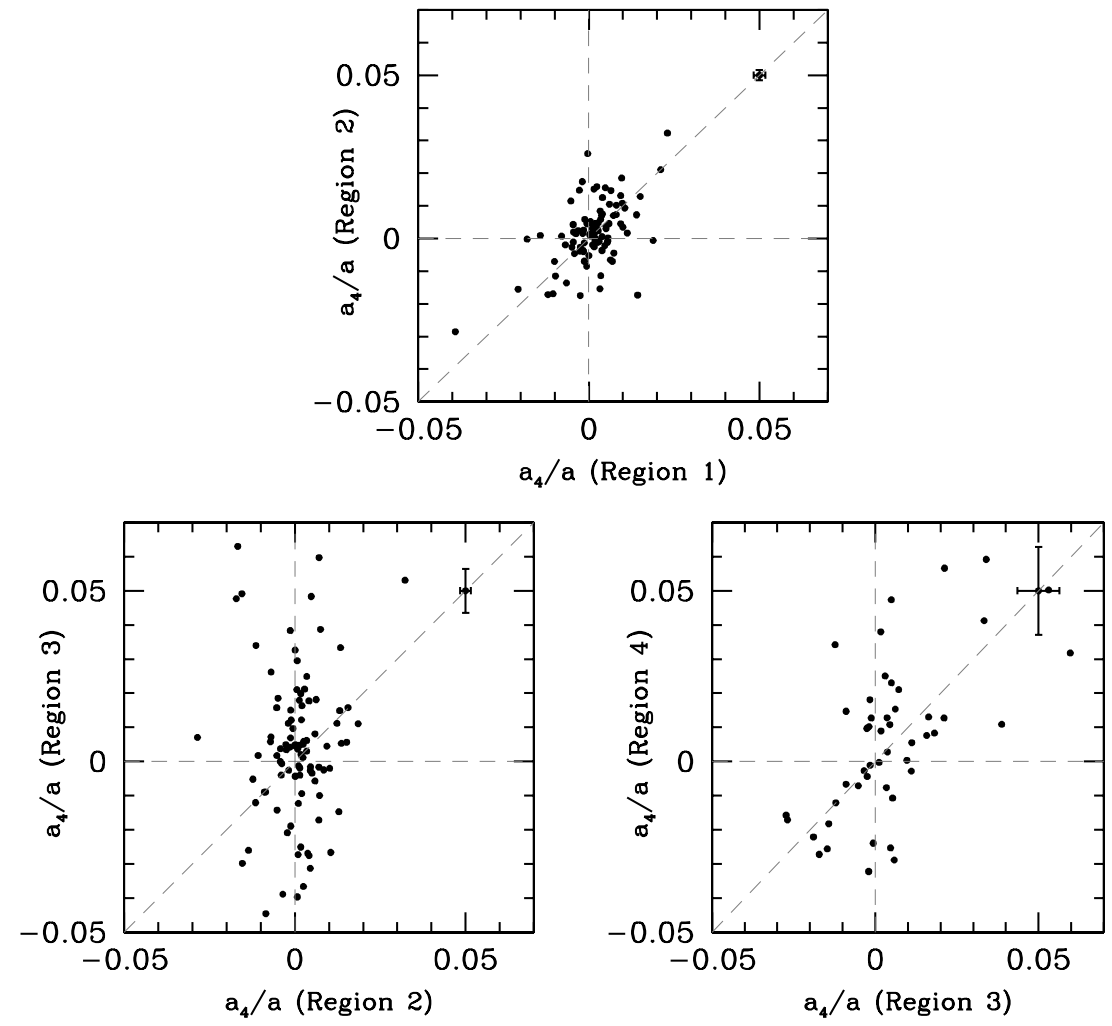

Figure 9. Correlation of $a_{4} / a$ parameters from neighboring regions. The error bars are as in Figure 8

\subsection{Are Distribution of Isophotal Parameters Statistically Different in Different Regions?}

We performed a two-sample Kolmogorov-Smirnov test (K-S test) to investigate whether the distribution of $a_{4} / a, a_{3} / a$, and $\epsilon$ are the same in our four regions (see Tables 5-7).
We used AstroStat, ${ }^{8}$ which uses a public domain statistical computing package $R^{9}$ for statistical analysis, to perform the K$\mathrm{S}$ test, in which the null hypothesis that the two samples belong

\footnotetext{
8 http://vo.iucaa.ernet.in/ voi/AstroStat.html

9 http://www.r-project.org/
} 
Table 6

Results of a Two Sample K-S Test for the $a_{3} / a$ Parameter $^{\mathrm{a}}$

\begin{tabular}{|c|c|c|c|c|c|c|c|c|}
\hline \multirow[t]{2}{*}{ Sample } & \multicolumn{2}{|c|}{ Region 1} & \multicolumn{2}{|c|}{ Region 2} & \multicolumn{2}{|c|}{ Region 3} & \multicolumn{2}{|c|}{ Region 4} \\
\hline & $D$ & $P$ & $D$ & $P$ & $D$ & $P$ & $D$ & $P$ \\
\hline Hao et al. (2006a) & 0.30 & $2.85 \mathrm{e}-07$ & 0.30 & $1.65 \mathrm{e}-09$ & 0.33 & $1.16 \mathrm{e}-08$ & 0.44 & $1.34 \mathrm{e}-08$ \\
\hline Region 1 & $\ldots$ & $\ldots$ & 0.10 & 0.613 & 0.21 & 0.031 & 0.29 & 0.007 \\
\hline Region 2 & $\ldots$ & $\cdots$ & $\ldots$ & $\ldots$ & 0.19 & 0.034 & 0.28 & 0.005 \\
\hline Region 3 & $\ldots$ & $\ldots$ & $\ldots$ & $\ldots$ & $\ldots$ & $\ldots$ & 0.19 & 0.151 \\
\hline
\end{tabular}

Note. ${ }^{\text {a }}$ Notation as in Table 5.

Table 7

Results of a Two Sample K-S Test for the $(1-b / a)$ Parameter $^{\mathrm{a}}$

\begin{tabular}{|c|c|c|c|c|c|c|c|c|}
\hline \multirow[t]{2}{*}{ Sample } & \multicolumn{2}{|c|}{ Region 1} & \multicolumn{2}{|c|}{ Region 2} & \multicolumn{2}{|c|}{ Region 3} & \multicolumn{2}{|c|}{ Region 4} \\
\hline & $D$ & $P$ & $D$ & $P$ & $D$ & $P$ & $D$ & $P$ \\
\hline Hao et al. (2006a) & 0.28 & $3.31 \mathrm{e}-06$ & 0.28 & $3.93 \mathrm{e}-08$ & 0.20 & 0.002 & 0.14 & 0.30 \\
\hline Region 1 & $\ldots$ & $\ldots$ & 0.11 & 0.466 & 0.19 & 0.068 & 0.27 & 0.002 \\
\hline Region 2 & $\ldots$ & $\ldots$ & $\ldots$ & $\ldots$ & 0.16 & 0.098 & 0.23 & 0.039 \\
\hline Region 3 & $\ldots$ & $\ldots$ & $\cdots$ & $\cdots$ & $\ldots$ & $\cdots$ & 0.13 & 0.574 \\
\hline
\end{tabular}

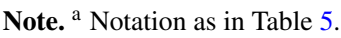

to the same parent distribution is tested. The strength of evidence in support of a null hypothesis is given by probability $P$ in the table. If the $P$ is less than the assumed level of significance, the hypothesis is rejected, where the level of significance is the probability of making a decision to reject the null hypothesis when the null hypothesis is true.

From the K-S test, we find that the distribution of $\epsilon$ in neighboring regions belongs to the same parent distribution, as the test is accepted at the 5\% level of significance. The distribution of $a_{4} / a$ and $a_{3} / a$ in Region 2 and Region 3 may not belong to the same parent distribution, as the test is rejected at the $5 \%$ level of significance. The distribution of the $a_{4} / a$ and $a_{3} / a$ parameters in Region 1 and Region 2 are from the same parent population, as suggested by the high $P$-value $(=0.86$ and $0.61)$ obtained from the K-S test. Similarly, the distribution of the $a_{4} / a$ and $a_{3} / a$ parameters in Region 3 and Region 4 are the same as the test is accepted at the $5 \%$ level of significance, though the strength of evidence in support of the null hypothesis is not as strong as it is for Region 1 and Region 2. The results of the K-S test clearly show the discontinuity in the distribution of parameters as we go from Region 2 to Region 3.

\subsection{Frequency of Boxy and Disky Ellipticals}

Bender et al. (1989) found that $\sim 1 / 3$ of their sample galaxies show boxy isophotes, $\sim 1 / 3$ show pointed isophotes, and $\sim 1 / 3$ of isophotes have a deviation smaller than $0.2 \%$ of the semimajor axis length. The frequency of boxy and disky isophotes in different radial regions of our sample galaxies is given in Table 8 . We find a larger fraction of disky isophotes in Regions 1 and 2 . The diskiness is generally attributed to the presence of a weak edge-on disk superposed on the spheroidal main body. One of the possible reasons for the increased fraction of disky isophotes in Region 1 is detection of those weak disks in our sample galaxies which would not be detected with relatively low $\mathrm{S} / \mathrm{N}$ images.

The major fraction of our galaxies have either boxy or disky isophotes in their outer regions: we find that the fraction of near-circular galaxies having $\left|a_{4} / a\right| \leqslant 0.2 \%$ is less than $10 \%$ in Regions 3 and 4, compared with 25\%-30\% in Regions 1 and 2. This is consistent with the expectation that tidal extensions and other environmental effects are likely to be stronger in the outer regions as compared to the inner regions of galaxies.

We also find a higher frequency of boxy as compared to disky isophotes in the outer regions. It has been suggested by Nieto $\&$ Bender (1989) that tidal extensions may also cause pointed isophotes. The higher fraction of boxy isophotes indicates (1) the presence of additional forces along with the tidal extensions; and (2) that such forces are either more frequent or more effective in outer regions.

The frequency of disky isophotes is higher in the outer regions of dwarf early-type galaxies having $M_{\mathrm{B}}>17.0$ in our sample. The fractions of disky isophotes for such galaxies in Regions $1-4$ are $1 / 6,4 / 8,4 / 6$, and $2 / 2$, respectively. The denominators of above fractions for each region are the total number of dwarf early-type galaxies for which isophotal parameters are available. This higher fraction of disky isophotes in outer regions from Region 1 to Region 4 observed for dwarf early-type galaxies in our sample is quite different from the corresponding frequency in different regions when we consider all of the galaxies in the sample. Differences in the environment of the dwarf and luminous early-type galaxies may explain the observed differences of their isophotal properties, as dwarf elliptical galaxies are found in high-density regions, either in galaxy clusters or in locations near more massive spiral and elliptical galaxies (Ferguson \& Sandage 1989; Van Zee et al. 2004).

\subsection{Correlation Between Isophotal Shape and Ellipticity}

Figure 10 shows plots of $a_{4} / a$ against ellipticity $\epsilon$ for galaxies in each of our four radial regions. Our data are most complete for Region 2; we miss some galaxies in Region 1 when the "seeing" $r_{s}>1.5 R_{50}$, and from Regions 3 and 4 due to confusion and low $\mathrm{S} / \mathrm{N}$. The solid lines in each panel define a chevron-shaped region within which all galaxies from the sample of Bender et al. (1989) were found. The dotted lines are extrapolations of these lines to higher values of ellipticity. 
Table 8

Frequency of Boxy and Disky Isophotes

\begin{tabular}{llcccc}
\hline \hline Radial Bin & Region & $\begin{array}{c}\text { Number of Galaxies } \\
\text { with Data in Region }\end{array}$ & Boxy $(\%)$ & Disky $(\%)$ & $\left|a_{4} / a\right| \leqslant 0.2 \%(\%)$ \\
\hline$r_{s}-1.5 R_{50}$ & Region 1 & 96 & 25 & 48 & 27 \\
\hline $1.5 R_{50}-3.0 R_{50}$ & Region 2 & 131 & 28 & 43 & 29 \\
\hline $3.0 R_{50}-4.5 R_{50}$ & Region 3 & 96 & 55 & 36 & 09 \\
\hline$r>4.5 R_{50}$ & Region 4 & 52 & 54 & 40 & 06 \\
\hline
\end{tabular}

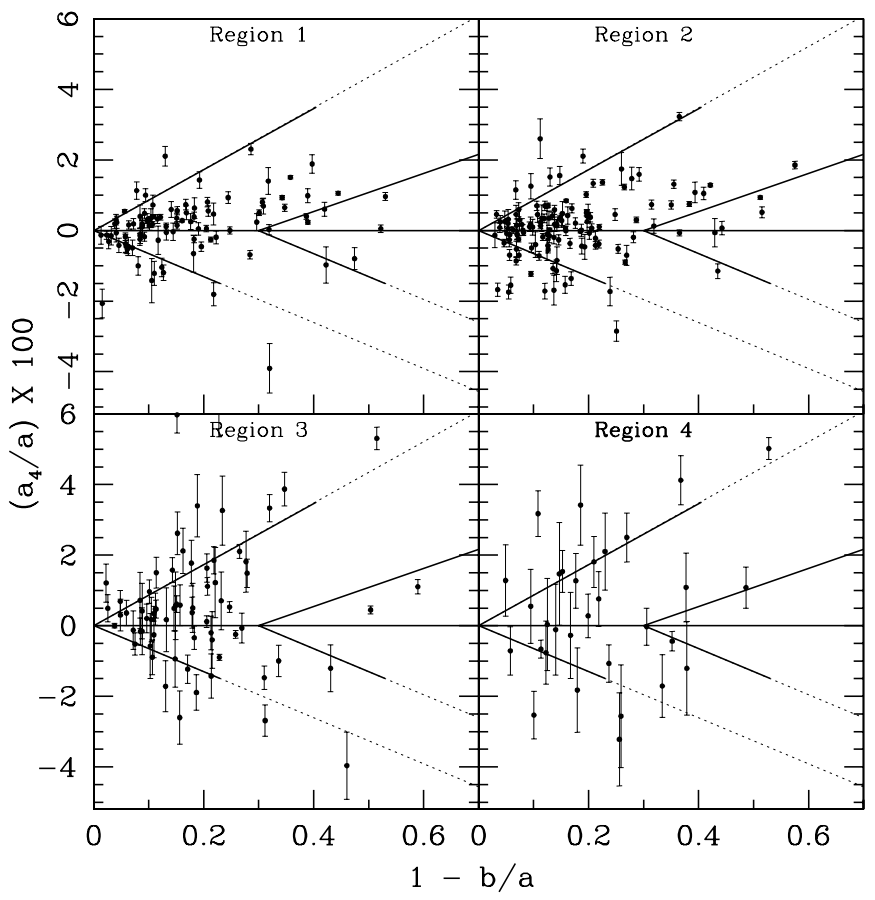

Figure 10. Isophote shape parameter $a_{4} / a$ plotted against ellipticity in the four radial regions. In Regions 3 and 4, we omit galaxies with errors $\sigma(a 4 / a)>0.01$ and 0.015, respectively, for clarity. Bender et al. (1989) found that their galaxies lay within the chevron-shaped region between the pairs of solid diagonal lines.

The distributions in Regions 1 and 2 are similar to each other, and most galaxies lie within the zone defined by Bender et al. (1989). Moreover, our galaxies show the same trend, with a preponderance of disky isophotes (with positive $a_{4} / a$ ) which extend to larger values as the ellipticity increases. However, we do find some galaxies lying outside Bender et al.'s (1989) boundaries, in particular, a group with boxy isophotes (negative $a_{4} / a$ ) and low ellipticity in Region 2 . We also find some galaxies with large ellipticity $(1-b / a>0.4)$ but with $a_{4} / a$ near zero.

Our data become increasingly incomplete and with larger errors in Regions 3 and 4, where we have plotted only points with errors less than 0.01 and 0.015 , respectively. The pattern appears similar in the two lower panels of Figure 10, and different from the pattern in the inner regions, although this may be at least partly due to the increasing errors and smaller samples.

Overall, the isophote shape data are consistent with the patterns seen in Section 4.4 and clearly indicate that the phenomena responsible for keeping the isophotes close to an elliptical shape at small radii (of the rounder galaxies) weaken as we go further out along the radius. Another possibility is that the effects of tidal interaction become more dominant, to make the isophotes deviate from the elliptical shape. Environmental studies may show whether this is true.

\section{DISCUSSION}

\subsection{Are Projection Effects Important?}

The results obtained by Bender et al. (1989) indicate beyond doubt that the boxiness or diskiness is an intrinsic property of the early-type galaxies rather than an effect caused by projection. This means that the sign of the $a_{4} / a$ parameter is independent of projection effects but it is not possible to say how the absolute value of $a_{4} / a$ depends on the viewing angle. The trend found by Bender et al. (1989) and Hao et al. (2006a), that the larger values of $\left|a_{4} / a\right|$ are observed for galaxies which appear more elliptical is consistent, with a few exceptions, with the results obtained for our sample galaxies in Section 4.5.

\subsection{Comparison with $N$-body Simulations}

The differences in the properties of boxy and disky elliptical are also supported by the $N$-body simulations of major mergers (e.g., Hernquist 1993; Lima Neto \& Combes 1995; Khochfar \& Burkert 2005) but some of the findings of $N$-body simulations are not consistent with the observations. For example, Stiavelli et al. (1991) and Heyl et al. (1994) analyzed the isophotal shapes of the remnants from their $N$-body simulations of dissipationless collapse and found that the same object has a value of $a_{4}$ negative or positive, depending on the viewing angle. Similarly, Governato et al. (1993) have reported that isophotal shape cannot discriminate between the possible origin of the early-type galaxies, at least in the range $-1 \leqslant a_{4} / a \times 100 \leqslant 1$, because of the dependence of the shape upon the viewing angle as seen using simulations. We do not understand the dependence of the $a_{4} / a$ parameter on viewing angle found by the above authors, which is inconsistent with the observations. In the future, the understanding of true intrinsic shapes of early-type galaxies may elucidate the effect of projection on the value of the $a_{4} / a$ parameter.

$N$-body merger simulations have been widely used by various researchers to study the origin of boxy and disky ellipticals. Simulations have been done taking progenitors for the merger as (1) a combination of galaxies with different morphologies and (2) a combination of galaxies with different mass ratios. Bournaud et al. (2005) have shown in their Figure 2, that a 7:1 merger produces a galaxy with boxy-isophotes in the inner region, while the outer region is highly disky. Such radial variation has also been reported using the analysis of $2 \mathrm{MASS}$ data for the Arp mergers (see Chitre \& Jog 2002, the Appendix). Similar merger scenarios can be considered as the possible origin of the inner boxy and outer disky isophotes observed in some of our sample galaxies.

Bournaud et al. (2005) have shown that for a merger with a 7:1 mass ratio, outer diskiness is observed for a 25 mag $\operatorname{arcsec}^{-2}$ isophote around $20 \mathrm{kpc}$ from the center (see their Figures 2 and 3 ). In the region $\sim 20-30 \mathrm{kpc}$, the surface density falls by a factor of $\sim 80-100$, and for a constant mass-to-light ratio, this 


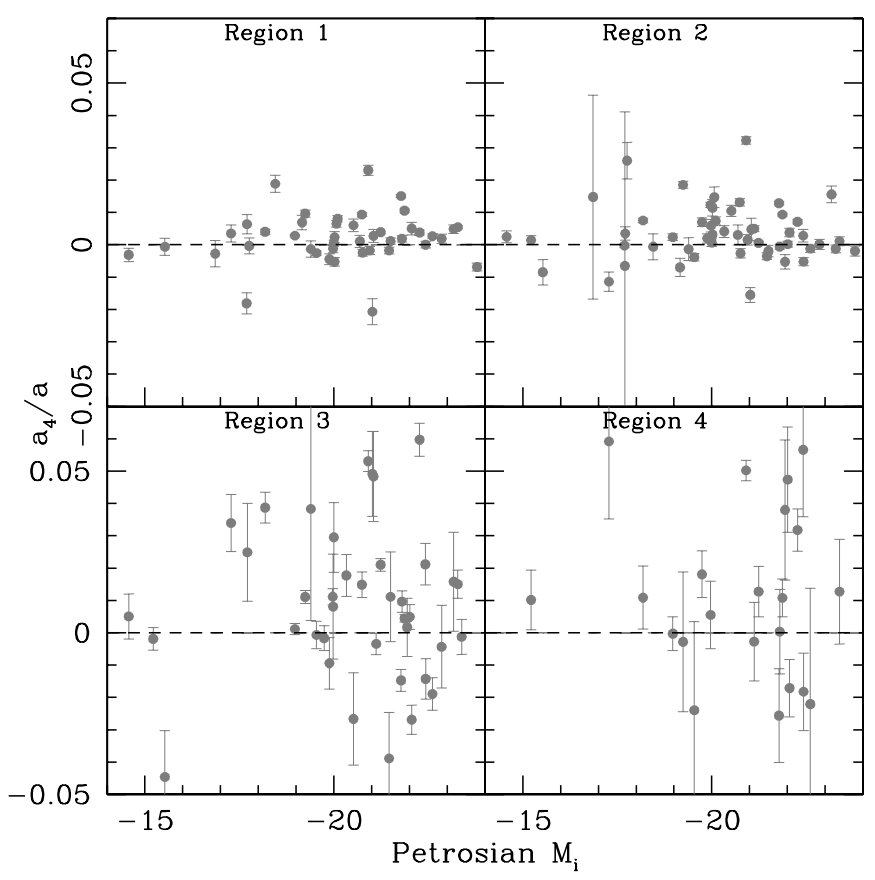

Figure 11. Isophotal shape parameter $a_{4} / a$ plotted against absolute Petrosian $i$ magnitude.

corresponds to a $\sim 4-5$ mag difference. The outer region of Bournaud et al. (2005) corresponds to Region 4 of most of our sample galaxies, and thus a disk is clearly indicated at such large radii.

A typical value of $a_{4} / a \simeq 0.01-0.02$ is obtained for the remnants of collisionless $N$-body simulations of binary mergers of disk galaxies with mass ratios of $1: 1,2: 1,3: 1$, and $4: 1$ (see Nabb \& Burkert 2003, Figure 3), while observations show larger diskiness in early-type galaxies. For example, observed values $a_{4} / a \lesssim 0.03$ are obtained for the galaxies studied by Bender et al. (1989), while some of our sample galaxies show larger diskiness $\left(a_{4} / a>0.03\right)$. Such galaxies are also a part of the sample studied by Hao et al. (2006b, Figure 1). The origin of strong diskiness therefore should have a different merger scenario from that studied by Nabb \& Burkert (2003). The galaxies with larger diskiness could well be explained by hybrid mergers, in the mass ratio range of 4:1-10:1 (Bournaud et al. 2004 , 2005). They report $a_{4} / a=0.064 \pm 0.01$ for mergers with mass ratio $4.5: 1$ and $a_{4} / a \simeq 0.07$ for mergers with mass ratio $7: 1$.

In binary merger scenarios, disky ellipticals are produced by 3:1-4:1 mergers, while boxy ellipticals are a product of equal-mass 1:1 mergers (Bournaud et al. 2007). But equalmass binary mergers fail to reproduce the most boxy elliptical galaxies, particularly giant ellipticals (Nabb \& Burkert 2003; Nabb \& Ostriker 2009). Alternatively, multiple minor mergers can reproduce boxy ellipticals, where the product of mergers mainly depends on the total merged mass. When total merged mass increases $(\geqslant 1.6)$, on average, the remnants tend to show boxy isophotes (see Bournaud et al. 2007, Figure 5). The merged mass has been defined in a particular way by authors in their paper: for instance, when the initial galaxy has merged with three companions, each of them having a 5:1 mass ratio, this so-called merged mass is 1.6 (1.0 for the main initial galaxy and 0.2 for each companion). Figure 11 shows a plot of $a_{4} / a$ with absolute $i$-band magnitude for the galaxies with redshift information. We find that a larger number of galaxies with boxiness have
$M_{i}<-19.5$, in all regions along the radius. Since more luminous galaxies will be more massive, the above trend can be considered similar to the trend observed for boxiness of the remnants of multiple minor mergers observed by Bournaud et al. (2007), though we do not exactly know the mass corresponding to $M_{i}<-19.5$.

We have tried to compare our observations with the isophotal shapes of the merger remnants but it is not in the scope of this paper to trace the merger history of the sample galaxies on the basis of observed isophotal shapes.

\section{CONCLUSIONS}

In this paper, we have studied the properties of the isophotal shapes of early-type galaxies (E/SO) to very faint outer regions, well beyond the levels reached by previous studies of this type. Our study is based on deep five-color Hale Telescope LFC CCD images of several fields, originally taken for a different project, that enable us to reach a SBs some 4 mag $\operatorname{arcsec}^{-2}$ fainter than most similar studies. Here, we present the first results for one $14^{\prime} \times 25^{\prime}$ field.

We selected target galaxies using the deep LFC $i$-band image of the field and used much shallower SDSS imaging data to get basic parameters, such as apparent magnitudes and Petrosian radii, for each galaxy. 266 sufficiently large and bright galaxies were selected for further study, 132 of which were identified as being of early-type on the basis of their bulge-to-total light ratios. We obtained spectra for over half of this sample using the multifiber system AAOmega on the AAT. These yielded reliable redshifts for 53 of the early-type galaxies, enabling us to derive their absolute magnitudes and physical sizes.

We fitted a sequence of ellipses to successive isophotes in the deep LFC images and derived a range of isophotal shape parameters that measure their ellipticity and orientation, and also higher order departures from a purely elliptical shape. We then derive mean values for these parameters in four radial bins along the semi-major axis of each galaxy. We find empirical fitting formulae for the probability distribution of the different isophotal parameters in each bin, which will be useful for comparison with theoretical studies, e.g., from $\mathrm{N}$-body simulations.

Finally, we have investigated possible correlations of isophotal shape parameters with other global properties of the galaxies, and inspected whether the correlations change along the radius. We find that the isophotal shapes of the inner regions of our sample of galaxies are statistically different from the isophotal shapes observed in the outer regions. In the central regions, we see patterns similar to those seen in previous studies of nearby galaxies, with some galaxies showing "boxy" isophotes while others appear "disky." However, the pattern seen in the inner region of each galaxy tends to change as the radius increases, suggesting that while the inner parts of the galaxies are coherent and presumably the result of specific dynamical processes, at larger radii, the shapes and orientations of the isophotes change and the behavior is not well defined. This may indicate effects from the formation and evolution of each galaxy which are not yet fully relaxed. However, for a full analysis of these data we need to know the distances to the galaxies so that we can derive their luminosities, actual sizes, and other physical parameters. We hope to obtain fiber spectra for many more galaxies to do this, preferably with one of the new Integral Field Unit systems that would avoid the effects of "aperture bias" that arise in single-fiber spectra, and enable us to compare galaxies at very different redshifts. 
Two of the authors (L.C. and S.K.P.) are grateful to ISRO for providing funds for this project under their RESPOND scheme (project no. ISRO/RES/2/343/2007-08). A.M. was supported by NSF grants AST-0407488 and AST-0909182. This paper makes use of deep LFC data obtained at the California Institute of Technology's Palomar Observatories and we acknowledge the role of S.G. Djorgovski, M. Bogosavljevic, and E. Glikman that resulted in the data Facility: Hale Telescope. We are thankful to Professor Chanda Jog for giving valuable suggestions through email communication regarding comparison of our work with results from $N$-body simulations.

We thank the anonymous referee for valuable comments.

We thank the staff of the Australian Astronomical Observatory for obtaining the AAT AAOmega spectra for us during Service Observing.

Funding for the Sloan Digital Sky Survey (SDSS) and SDSS-II has been provided by the Alfred P. Sloan Foundation, participating institutions, the National Science Foundation, the U.S. Department of Energy, the National Aeronautics and Space Administration, the Japanese Monbukagakusho, the Max Planck Society, and the Higher Education Funding Council for England. The SDSS Web site is http://www.sdss.org/.

The SDSS is managed by the Astrophysical Research Consortium (ARC) for participating institutions. The participating institutions are the American Museum of Natural History, Astrophysical Institute Potsdam, University of Basel, University of Cambridge, Case Western Reserve University, The University of Chicago, Drexel University, Fermilab, the Institute for Advanced Study, the Japan Participation Group, The Johns Hopkins University, the Joint Institute for Nuclear Astrophysics, the Kavli Institute for Particle Astrophysics and Cosmology, the Korean Scientist Group, the Chinese Academy of Sciences (LAMOST), Los Alamos National Laboratory, the Max-PlanckInstitute for Astronomy (MPIA), the Max-Planck-Institute for Astrophysics (MPA), New Mexico State University, Ohio State University, University of Pittsburgh, University of Portsmouth, Princeton University, the United States Naval Observatory, and the University of Washington.

\section{APPENDIX}

\section{ESTIMATION OF SURFACE BRIGHTNESS LIMIT WITH LFC IMAGE AS COMPARED TO THE SDSS IMAGE}

Following is the estimation of how far in the SB we can go with LFC images as compared to SDSS images assuming the Poisson statistics.

The number of source and background photons collected in $t$ seconds using a telescope of diameter $D$ is

$$
\begin{aligned}
& N_{s}=n_{s} D^{2} t, \\
& N_{b}=n_{b} D^{2} t,
\end{aligned}
$$

where $n_{s}$ and $n_{b}$ are the rate of photons received in unit area from a source and the background. Signal-to-noise ratio for an extended source which spans a solid angle $\Omega$ can be given as

$$
\frac{N_{s}}{\sigma_{s}}=\frac{n_{s}\left(\Omega D^{2} t\right)^{\frac{1}{2}}}{\sqrt{n_{s}+2 n_{b}}} .
$$

For $n_{s} \ll n_{b}$, the above equation becomes

$$
\frac{N_{s}}{\sigma_{s}}=\frac{n_{s}\left(\Omega D^{2} t\right)^{\frac{1}{2}}}{\sqrt{2 n_{b}}} .
$$

The $\mathrm{S} / \mathrm{N}$ will increase by factor of $\sqrt{P}$, if the source intensity is obtained by averaging the intensity in a number of pixels $P$,

$$
\mathrm{S} / \mathrm{N}_{\mathrm{av}}=\frac{N_{s} \sqrt{P}}{\sigma_{s}} .
$$

In the ellipse fitting task, the average intensity of an isophote is averaged around elliptical annuli between the fitted ellipses. The output table of ellipse gives parameters INTENS and INT_ERR, which are the mean intensity along the fitted ellipse and the total error in the intensity. We can write

$$
\frac{\text { INTENS }}{\text { INT_ERR }}=\frac{N_{s} \sqrt{P}}{\sigma_{s}} .
$$

If the isophotes in two different images have the same INTENS/INT_ERR, then

$$
\frac{n_{s 1}\left(P_{1} \Omega_{1} D_{1}^{2} t_{1}\right)^{\frac{1}{2}}}{\sqrt{2 n_{b 1}}}=\frac{n_{s 2}\left(P_{2} \Omega_{2} D_{2}^{2} t_{2}\right)^{\frac{1}{2}}}{\sqrt{2 n_{b 2}}} .
$$

The difference in SB of isophotes $\left(\mu_{s 1}-\mu_{s 2}\right)$ with the same INTENS/INT_ERR in two different images can be given as

$$
\begin{aligned}
\mu_{s 1}-\mu_{s 2}= & \frac{1}{2}\left[\mu_{b 1}-\mu_{b 2}-2.5 \log \left[\left(\frac{D_{2}}{D_{1}}\right)^{2}\left(\frac{t_{2}}{t_{1}}\right)\right.\right. \\
& \left.\left.\times\left(\frac{\Omega_{2}}{\Omega_{1}}\right)\left(\frac{P_{2}}{P_{1}}\right)\right]\right] .
\end{aligned}
$$

The ellipse is fitted till INTENS/INT_ERR drops to three to derive SB profiles in the images of SDSS and LFC (see Figure 1 as an example). Putting in values for the SDSS image: $D_{1}=$ $2.5 \mathrm{~m}, t_{1}=54 \mathrm{~s}, P_{1}=72$ pixels, $\mu_{b 1}=20.3 \mathrm{mag} \mathrm{arcsec}^{-2}$ and for the LFC image: $D_{1}=5 \mathrm{~m}, t_{1}=9065 \mathrm{~s}, P_{1}=184$ pixels, $\mu_{b 1}=20.2 \mathrm{mag} \operatorname{arcsec}^{-2}$, in Equation (A7), we expect reach a minimum of $\sim 4 \mathrm{mag} \operatorname{arcsec}^{-2}$ deeper in the LFC image as compared to the SDSS image.

\section{REFERENCES}

Abazajian, K. N., Adelman-McCarthy, J. K., Agüeros, M. A., et al. 2009, ApJS, 182,543

Bailey, J. A., et al. 2004, The 2DFDR Data Reduction System Users Manual (Coonabarabran, Australia: AAO) (http://www.aao.gov.au/AAO/2dF/ manual.html)

Bender, R., Döbereiner, S., \& Möllenhoff, C. 1988, A\&AS, 74, 385

Bender, R., Surma, P., Döbereiner, S., Mollenhoff, C., \& Madejsky, R. 1989, A\&A, 217, 35

Bertin, E., \& Arnouts, S. 1996, A\&AS, 117, 393

Blanton, M. R., \& Roweis, S. 2007, AJ, 133, 734

Blanton, M. R., Schlegel, D. J., Strauss, M. A., et al. 2005, AJ, 129, 2562

Bournaud, F., Combes, F., \& Jog, C. J. 2004, A\&A, 418, 27

Bournaud, F., Jog, C. J., \& Combes, F. 2005, A\&A, 437, 69

Bournaud, F., Jog, C. J., \& Combes, F. 2007, A\&A, 476, 1179

Busko, I. C. 1996, in ASP Conf. Ser. 101, Astronomical Data Analysis Software and Systems V, ed. G. H. Jacoby \& J. Barnes (San Francisco, CA: ASP), 139

Cannon, R. D., Drinkwater, M., Edge, A., et al. 2006, MNRAS, 372, 425

Capaccioli, M., Piotto, G., \& Rampazzo, R. 1988, AJ, 96, 487

Chitre, A., \& Jog, C. J. 2002, A\&A, 388, 407

Ferguson, H. C., \& Sandage, A. 1989, ApJL, 346, L53

Ferrarese, L., van den Bosch, F. C., Ford, H. C., Jaffe, W., \& O’Connell, R. W. 1994, AJ, 108, 1598

Freeman, K. C. 1970, ApJ, 160, 811

Governato, G., Reduzzi, L., \& Rampazzo, R. 1993, MNRAS, 261, 379 
Hao, C. N., Mao, S., Deng, Z. G., Xia, X. Y., \& Wu, H. 2006a, MNRAS, 370,1339

Hao, C. N., Mao, S., Deng, Z. G., Xia, X. Y., \& Wu, H. 2006b, MNRAS, 373,1264

Hernquist, L. 1993, ApJ, 409, 548

Heyl, J. S., Hernquist, L., \& Spergel, D. N. 1994, ApJ, 427, 165

Jedrzejewski, R. 1987, MNRAS, 226, 747

Jog, C. J., \& Maybhate, A. 2006, MNRAS, 370, 891

Khochfar, S., \& Burkert, A. 2005, MNRAS, 359, 1379

Lauer, T. R. 2005, AJ, 129, 2138

Lima Neto, G. B., \& Combes, F. 1995, A\&A, 294, 657

Mahabal, A., Stern, D., Bogosavljević, M., Djorgovski, S. G., \& Thompson, D. 2005, ApJL, 634, L9

Malin, D., \& Carter, D. 1980, Natur, 285, 643

Milvang-Jensen, B., \& Jørgensen, I. 1999, BaltA, 8, 535

Miszalski, B., Shortridge, K., Saunders, W., Parker, Q. A., \& Croom, S. M. 2006, MNRAS, 371, 1537

Naab, T., \& Burkert, A. 2003, ApJ, 597, 893

Naab, T., Burkert, A., \& Hernquist, L. 1999, ApJL, 523, L133

Naab, T., Khochfar, S., \& Burkert, A. 2006, ApJL, 636, L81

Naab, T., \& Ostriker, J. P. 2009, ApJ, 690, 1452
Nieto, J. L., \& Bender, R. 1989, A\&A, 215, 266

Pasquali, A., van den Bosch, F. C., \& Rix, H.-W. 2007, ApJ, 664, 738

Peng, C. Y., Ho, L. C., Impey, C. D., \& Rix, H.-W. 2002, AJ, 124, 266

Poggianti, B. M. 1997, A\&AS, 122, 399

Press, W. H., Teukolsky, S. A., Vetterling, W. T., \& Flannery, B. P. 1992, Numerical Recipes in Fortran (Cambridge: Cambridge Univ. Press), 614

Quinn, P. 1984, ApJ, 279, 596

Rawat, A., Kembhavi, A. K., Hammer, F., Flores, H., \& Barway, S. 2007, A\&A, 496, 483

Rest, A., van den Bosch, F. C., Jaffe, W., et al. 2001, AJ, 121, 2431

Ryden, B. S., Forbes, D. A., \& Terlevich, A. I. 2001, MNRAS, 326, 1141

Saunders, W., Bridges, T., Gillingham, P., et al. 2004, Proc. SPIE, 5492, 389

Schlegel, D. J., Finkbeiner, D. P., \& Davis, M. 1998, ApJ, 500, 525

Sersic, J. L. 1968, Atlas de Galaxias Australes (Cordoba: Obs. Astron, Univ. Nac. Ćordoba)

Smith, J. A., Tucker, D. L., Kent, S., et al. 2002, AJ, 123, 2121

Stiavelli, M., Londrillo, P., \& Messina, A. 1991, MNRAS, 251, 57

Tremblay, G. R., Chiaberge, M., Donzelli, C. J., et al. 2007, ApJ, 666, 109

van den Bosch, F. C. 1994, AJ, 108, 1579

Van Zee, L., Skillman, E. D., \& Haynes, M. P. 2004, AJ, 128, 121 